\title{
THE FIRST STARS: A LOW-MASS FORMATION MODE
}

\author{
Athena STacy ${ }^{1,2}$ and Volker Bromm ${ }^{3}$ \\ ${ }^{1}$ NASA Goddard Space Flight Center, Greenbelt, MD 20771, USA; athena.stacy@berkeley.edu \\ ${ }^{2}$ University of California, Berkeley, CA 94720 , USA \\ ${ }^{3}$ Department of Astronomy and Texas Cosmology Center, University of Texas, Austin, TX 78712, USA \\ Received 2013 July 6; accepted 2014 February 19; published 2014 March 27
}

\begin{abstract}
We perform numerical simulations of the growth of a Population III stellar system under photodissociating feedback. We start from cosmological initial conditions at $z=100$, self-consistently following the formation of a minihalo at $z=15$ and the subsequent collapse of its central gas to high densities. The simulations resolve scales as small as $\sim 1$ AU, corresponding to gas densities of $10^{16} \mathrm{~cm}^{-3}$. Using sink particles to represent the growing protostars, we evolve the stellar system for the next $5000 \mathrm{yr}$. We find that this emerging stellar group accretes at an unusually low rate compared with minihalos which form at earlier times $(z=20-30)$, or with lower baryonic angular momentum. The stars in this unusual system will likely reach masses ranging from $<1 M_{\odot}$ to $\sim 5 M_{\odot}$ by the end of their main-sequence lifetimes, placing them in the mass range for which stars will undergo an asymptotic giant branch (AGB) phase. Based upon the simulation, we predict the rare existence of Population III stars that have survived to the present day and have been enriched by mass overflow from a previous AGB companion.
\end{abstract}

Key words: cosmology: theory - dark ages, reionization, first stars - stars: formation - stars: Population III

Online-only material: color figures

\section{INTRODUCTION}

Following the emission of the cosmic microwave background, the universe entered a period referred to as the "dark ages," when no luminous objects had yet formed. During this time, self-gravitating dark matter (DM) halos gradually grew in mass through the process of hierarchical merging. The dark ages ended when first stars, also known as Population III (Pop III), formed at $z \gtrsim 20$ within DM minihalos of mass $\sim 10^{6} M_{\odot}$ (e.g., Haiman et al. 1996; Tegmark et al. 1997; Yoshida et al. 2003).

The typical mass of Pop III stars remains an open question that is crucial to our understanding of the evolution of the early universe (Bromm 2013). Their mass determines the rate at which they emitted ionizing radiation, and thus the extent to which they contributed to the reionization of the universe (e.g., Kitayama et al. 2004; Sokasian et al. 2004; Whalen et al. 2004; Alvarez et al. 2006; Johnson et al. 2007). In addition, the Pop III mass determines how much they contributed to the metallicity of the intergalactic medium (IGM; Madau et al. 2001; Mori et al. 2002; Bromm et al. 2003; Wada \& Venkatesan 2003; Norman et al. 2004; Tornatore et al. 2007; Greif et al. 2007, 2010; Wise \& Abel 2008; Wise et al. 2012; Maio et al. 2011; recently reviewed in Karlsson et al. 2013). For instance, Pop III stars with main sequence masses in the range $40 M_{\odot}<$ $M_{*}<140 M_{\odot}$ or $M_{*}>260 M_{\odot}$ are expected to collapse directly into black holes, therefore releasing virtually no metals into the IGM. On the other hand, stars with mass $140 M_{\odot}<$ $M_{*}<260 M_{\odot}$ are predicted to explode as pair-instability supernovae (PISNe; Heger \& Woosley 2002), thereby releasing the entirety of their metal content into the IGM and surrounding halos. We furthermore note recent work which has found that stellar rotation may significantly lower the PISN mass range (Chatzopoulos \& Wheeler 2012; Yoon et al. 2012). Primordial stars within the range $15 M_{\odot}<M_{*}<40 M_{\odot}$ will end their lives as core-collapse $\mathrm{SNe}$, or possibly hypernovae in the case of rapid rotation (e.g., Nomoto et al. 2003). Constraining the initial mass of Pop III stars is therefore central to understanding how the radiation and metallicity they released affected the formation of later stellar generations.

Earlier work predicted that Pop III stars would form as single stars and grow to be very massive $\left(\gtrsim 100 M_{\odot}\right.$; e.g., Abel et al. 2002; Bromm et al. 2002; Bromm \& Loeb 2004; Yoshida et al. 2008). An analytical study by McKee \& Tan (2008) found that even when accounting for radiative feedback, a protostar can grow to greater than $100 M_{\odot}$. Though a portion of the inflow toward the protostar will be reversed due to a growing ionization front (I-front), this I-front will expand preferentially in directions perpendicular to the protostellar disk, while accretion through the disk can continue unimpeded until much later times.

In contrast to the above picture of single and massive Pop III stars, more recent work has shown that primordial gas will undergo fragmentation and develop into a disk within which a stellar multiple system will form (e.g., Clark et al. 2008, 2011a). Such fragmentation is seen in simulations even when initialized on cosmological scales (e.g., Turk et al. 2009; Stacy et al. 2010). Furthermore, Pop III multiplicity occurs down to very small scales $(\sim 10 \mathrm{AU})$ and in the majority of minihalos (Clark et al. 2011b; Greif et al. 2011), even when accounting for the effects of feedback from protostellar accretion luminosity (Smith et al. 2011; Stacy et al. 2012). Though a number of the above simulations exhibited disk fragmentation, the resolution study by Turk et al. (2012) found that increasing the number of resolution elements per Jeans mass leads to variation in gas morphology and suppression of disk formation. They did not follow subsequent protostellar accretion, however, so whether fragmentation instabilities might later develop could not be determined. Latif et al. (2013) performed a similar study but followed the gas evolution beyond the formation of the first peak to find that self-gravitating disks with very rapid accretion rates $\left(10^{-2} M_{\odot} \mathrm{yr}^{-1}\right)$ will indeed develop.

Several studies thus provide evidence that disk instability will develop after the first protostar arises in a primordial minihalo. In addition, a fraction of minihalos may also be subject to 
earlier fragmentation in a pre-collapse phase. Greif et al. (2013) find that this will lead to secondary clumps in at least two out of nine minihalos even before a protostar forms. Though these recent studies generally imply a broader IMF for Pop III stars, the predicted IMFs remain top-heavy. The primordial stellar systems still exhibit rapid accretion rates compared to modern-day star formation (e.g., Stacy \& Bromm 2013), and the most massive star in each system is still expected to eventually reach very high masses $\left(\gtrsim 10 M_{\odot}\right)$.

In this paper we present the first three-dimensional numerical simulation to follow the growth of a Pop III stellar system from cosmological initial conditions while also resolving nearly protostellar scales $\left(\sim 100 R_{\odot}\right)$ and accounting for the effects of photodissociating radiation. Recent work has found that $100 R_{\odot}$ $(\sim 1$ AU) is approximately the maximum radius reached by a Pop III protostar during its pre-main sequence evolution (see e.g., Hosokawa et al. 2010; Smith et al. 2012). Resolving these small scales corresponds to evolving the gas up to a maximum density of $10^{16} \mathrm{~cm}^{-3}$, at which point we continue the gas evolution for a further $5000 \mathrm{yr}$ by employing sink particles to represent the growing protostars. At this high density the gas is quickly approaching complete optical thickness to continuum radiation $\left(10^{18} \mathrm{~cm}^{-3}\right)$ and will not undergo further fragmentation on sub-sink scales (Yoshida et al. 2008). Our calculation therefore allows us to determine the true number of protostars that form within the central region of the host minihalo without missing any fragmentation due to lack of spatial resolution.

While our numerical feedback model is also able to follow the effects of ionizing radiation from a growing protostar (e.g., Greif et al. 2009), we find that the particular protostellar system we simulate does not contain stars sufficiently massive to produce an $\mathrm{H}$ II region. This is an unusual system with significant variation from the more typical rapidly accreting Pop III protostars studied in abovementioned work. Instead, the most massive star of the system considered here will most likely undergo an asymptotic giant branch (AGB) phase en route to becoming a white dwarf.

The AGB phase, which occurs for stars with initial masses between $\sim 0.8$ and $8 M_{\odot}$, plays an influential role in Galactic chemical evolution, so there is much interest in understanding the metal yields of low-metallicity AGB stars (e.g., Karakas 2010; Campbell \& Lattanzio 2008; Karakas \& Lugaro 2010). Similarly, if a significant population of AGB stars existed at high redshift, this would have consequences for metal and dust production in the early universe. Observations of highredshift $(z \gtrsim 6)$ galaxies and quasars indicate that significant amounts of dust had already formed at these early times, and the origin of such rapid dust production remains a subject of study (Bertoldi et al. 2003; Valiante et al. 2009; Cherchneff \& Dwek 2010; Gall et al. 2011). Though SNe were likely the major source of dust at these early times, Pop III AGB stars could have provided a significant contribution as well. This applies in particular to stars such as the three most massive ones we present from our simulation, predicted to reach $3-5 M_{\odot}$. These stars have sufficiently short main-sequence lifetimes $\left(\sim 10^{8} \mathrm{yr}\right)$ to undergo an AGB phase by $z=6$. In contrast, the smaller $1 M_{\odot}$ stars from our simulation are too long lived to provide any dust or metallicity contribution. The more massive AGB stars could also have significantly contributed to carbon and nitrogen production in the early universe, as well as s-process elements (Busso et al. 2001; Siess et al. 2002; Siess \& Goriely 2003).
Though much study of Pop III stars to date has emphasized the high-mass end of the Pop III IMF, we note that even some early studies predicted that typical Pop III stellar masses might be quite low, $\lesssim 1 M_{\odot}$. For instance, Kashlinsky \& Rees (1983) emphasized the importance of angular momentum in determining the mass of Pop III stars, predicting that rotational effects would cause the primordial gas clouds to collapse into a dense disk. Only after the disk cooled to $\sim 1000 \mathrm{~K}$ through $\mathrm{H}_{2}$ line emission could fragmentation occur. Nakamura \& Umemura (2001) predicted that fragmentation of primordial filaments would lead to a bimodal IMF, with a peak at $\sim 1 M_{\odot}$ as well as at $100 M_{\odot}$.

Here, we further explore the possible parameter space for Pop III star formation by modeling with high accuracy the growth of an unusually low-mass primordial system. Such cases are expected to be rare, since most Pop III systems have been found to contain one or more high-mass stars that likely dominated the overall Pop III impact on the IGM and later stellar generations. However, such low-mass stars as found in our simulation were potential survivors to the present-day, and may in principle be discovered within the Milky Way halo or nearby dwarf galaxies. In Section 2 we describe our numerical methodology, in Section 3 we discuss our protostellar evolution model, and in Section 4 we present our results. We discuss the impact of a global Lyman-Werner (LW) background in Section 5, and we conclude in Section 6.

\section{NUMERICAL METHODOLOGY}

\subsection{Initial Setup}

We carry out our investigation using GADGET-2, a widely tested three-dimensional $N$-body and smoothed particle hydrodynamics (SPH) code (Springel 2005). We begin with a $200 \mathrm{kpc}$ (comoving) box containing $128^{3}$ SPH gas particles and the same number of DM particles. The simulation is initialized at $z=100$. Positions and velocities are assigned to the particles in accordance with a $\Lambda$ CDM cosmology with $\Omega_{\Lambda}=0.7, \Omega_{\mathrm{M}}=0.3$, $\Omega_{\mathrm{B}}=0.04, \sigma_{8}=0.9$, and $h=0.7$. The gas and DM evolution is followed until the first minihalo forms and its central gas density reaches $10^{4} \mathrm{~cm}^{-3}$.

Once the site of the first minihalo is determined, the simulation is performed at higher resolution, starting again at $z=100$. The increased resolution is attained using a hierarchical zoomin procedure (e.g., Navarro \& White 1994; Tormen et al. 1997; Gao et al. 2005) in which four nested refinement boxes are placed within the cosmological box, centered on the site where the minihalo will eventually form. Within each refinement level, each particle from the lower level is replaced with eight "child" particles, so that in the most refined region the parent particle is replaced by 4096 child particles. The four refinement levels have lengths of 40, 35, 30, and $20 h^{-1} \mathrm{kpc}$ (comoving), so that the most highly refined level encompasses all the mass that will later be incorporated into the minihalo. The most refined SPH particles have mass $m_{\mathrm{SPH}}=5 \times 10^{-3} M_{\odot}$, and the resolution mass of the refined simulation is $M_{\text {res }} \simeq 1.5 N_{\text {neigh }} m_{\mathrm{SPH}} \lesssim 0.3 M_{\odot}$, where $N_{\text {neigh }} \simeq 40$ is the typical number of particles in the SPH smoothing kernel (e.g., Bate \& Burkert 1997).

\subsection{Cut-out Technique and Particle Splitting}

To increase computational efficiency, once the gas has reached densities of $10^{12} \mathrm{~cm}^{-3}$ we employ a "cut-out" technique in which all gas and DM beyond 3 pc from the densest gas 
particle is removed. At this point the central star-forming gas is gravitationally bound and under minimal influence from the mass of the outer minihalo and more distant regions of the cosmological box. The total mass of the cut-out is $3500 M_{\odot}$, and the minimum density is $\sim 10^{2} \mathrm{~cm}^{-3}$ (see, e.g., Stacy et al. 2012 for further details).

At the same time that we cut out the central $3 \mathrm{pc}$ of the cosmological box, we also further increase the particle resolution so that collapse to densities of $10^{16} \mathrm{~cm}^{-3}$ can be properly followed. We thus replace each SPH particle with eight child particles, each of which is placed randomly within the smoothing kernel of the parent particle. The mass of the parent particle is then evenly divided amongst the child particles. Each of these particles inherits the same chemical abundances, velocity, and entropy as the parent particle (see, e.g., Bromm \& Loeb 2003; Clark et al. 2011b). This ensures conservation of mass, internal energy, and linear momentum. After this process, each SPH particle in the new cut-out simulation has a mass of $m_{\mathrm{sph}}=6 \times 10^{-4} M_{\odot}$, and the new resolution mass is $M_{\text {res }} \simeq 0.03 M_{\odot}$. This final $M_{\text {res }}$ is close to the mass of the pressure-supported atomic core which develops once the opacity limit is reached (Yoshida et al. 2008), defining the point at which the protostar has first formed.

\subsection{Chemistry, Heating, and Cooling}

We utilize the same chemistry and thermal network as described in detail by Greif et al. (2009) and used in Stacy et al. (2012). In short, the code follows the abundance evolution of $\mathrm{H}, \mathrm{H}^{+}, \mathrm{H}^{-}, \mathrm{H}_{2}, \mathrm{H}_{2}^{+}, \mathrm{He}, \mathrm{He}^{+}, \mathrm{He}^{++}$, and $e^{-}$, as well as the three deuterium species $\mathrm{D}, \mathrm{D}^{+}$, and $\mathrm{HD}$. All relevant cooling mechanisms, including $\mathrm{H}_{2}$ collisions with $\mathrm{H}$ and $\mathrm{He}$ as well as other $\mathrm{H}_{2}$ molecules, are included. The thermal network also accounts for cooling through $\mathrm{H}_{2}$ collisions with protons and electrons, $\mathrm{H}$ and $\mathrm{He}$ collisional excitation and ionization, recombination, bremsstrahlung, and inverse Compton scattering.

Further $\mathrm{H}_{2}$ processes must also be included to properly model gas evolution to high densities. For instance, the chemistry and thermal network includes three-body $\mathrm{H}_{2}$ formation and the concomitant $\mathrm{H}_{2}$ formation heating, which become important at $n \gtrsim 10^{8} \mathrm{~cm}^{-3}$. Furthermore, when $n \gtrsim 10^{9} \mathrm{~cm}^{-3}$, cooling through $\mathrm{H}_{2}$ ro-vibrational lines becomes less effective as these lines grow optically thick. This is accounted for using an escape probability formalism together with the Sobolev approximation (see Yoshida et al. 2006; Greif et al. 2011 for further details). Simple fitting formulae are also available for estimating optically thick $\mathrm{H}_{2}$ rates (e.g., Ripamonti \& Abel 2004). However, Hirano \& Yoshida (2013) find that fitting formulae can overestimate the cooling rate, such as in cases when the gas has a substantial degree of rotation. This can lead to significant differences in gas evolution, such as accelerated gravitational collapse, when using fitting formulae as opposed to the more accurate Sobolev method.

The most important new process utilized in the thermal network is $\mathrm{H}_{2}$ collision-induced emission (CIE) cooling, which becomes significant when $n \gtrsim 10^{14} \mathrm{~cm}^{-3}$ (Frommhold 1994). As described in Greif et al. (2011), the reduction of the CIE cooling rate due to the effects of continuum opacity is handled through the following prescription (Ripamonti et al. 2002; Ripamonti \& Abel 2004):

$$
\Lambda_{\mathrm{CIE}, \text { thick }}=\Lambda_{\mathrm{CIE}, \text { thin }} \min \left(\frac{1-e^{-\tau_{\mathrm{CIE}}}}{\tau_{\mathrm{CIE}}}, 1\right)
$$

where

$$
\tau_{\mathrm{CIE}}=\left(\frac{n_{\mathrm{H}_{2}}}{7 \times 10^{15} \mathrm{~cm}^{-3}}\right)^{2.8}
$$

$\Lambda_{\mathrm{CIE} \text {,thin }}$ is the CIE cooling rate in the optically thin limit, and $\Lambda_{\mathrm{CIE} \text {,thick }}$ that in optically thick conditions. Hirano \& Yoshida (2013) compared gas evolution when continuum opacity effects are calculated using the above fitting formula, and when they are instead estimated using three-dimensional ray tracing. They find that when the fitting formula is used the gas collapses to $\sim 10^{17} \mathrm{~cm}^{-3}$ only slightly faster, by $\sim 1 \mathrm{yr}$. The differences in the thermal evolution are also minimal between the two methods. We thus expect the fitting formula above to be sufficiently accurate. However, while Hirano \& Yoshida (2013) modeled runaway gas collapse, it is possible that the differences would be more substantial when considering longer-term evolution of a disk, and this will be further examined in future work.

\subsection{Sink Particle Method}

When an SPH particle reaches a density of $n_{\max }=10^{16} \mathrm{~cm}^{-3}$, it is converted to a sink particle (e.g., Bate et al. 1995; Bromm et al. 2002; Martel et al. 2006; see also Stacy et al. 2012 for further details on the employed sink particle method.) The sink then grows in mass by accreting surrounding particles within its accretion radius, which we set equal to the resolution length such that $r_{\text {acc }}=L_{\text {res }} \simeq 1.0 \mathrm{AU}$.

The sink accretes a gas particle within $r_{\text {acc }}$ as long as the particle is not rotationally supported against infall onto the sink. This is determined by checking that the particle satisfies $j_{\mathrm{SPH}}<j_{\text {cent }}$, where $j_{\mathrm{SPH}}=v_{\text {rot }} d$ is the specific angular momentum of the gas particle, $j_{\text {cent }}=\sqrt{G M_{\text {sink }} r_{\text {acc }}}$ the level required for centrifugal support, and $v_{\text {rot }}$ and $d$ are the rotational velocity and distance of the particle relative to the sink. Particles that satisfy these criteria are removed from the simulation, and their mass is added to that of the sink. When the sink first forms it immediately accretes most of the particles within its smoothing length, so its initial mass is near the resolution mass of the simulation, $M_{\text {res }} \simeq 3 \times 10^{-2} M_{\odot}$. Its position and velocity are set to the average of that of the accreted particles.

These same accretion criteria are additionally used to determine whether two sinks may be merged. However, we note that modifications to the sink merging algorithm can significantly alter the sink accretion history (Greif et al. 2011). Recent work by Greif et al. (2012) has resolved sub-protostellar scales $\left(0.05 R_{\odot}\right)$ of primordial star-forming gas, tracking the merger rate of protostars by following their interactions without using sinks. They find that approximately half of the secondary protostars formed will indeed migrate toward and merge with the initial protostar. Our merging algorithm leads to a similar fraction of secondary sinks merging with the main sink. After the first sink arises, six secondaries later form, but two of them eventually merge with the initial sink. This roughly agrees with what Greif et al. (2012) find to occur on sub-sink scales.

\subsection{Ray-tracing Scheme}

Once the first sink particle forms, it represents a newly formed protostar and is used as the point source for modeling the effects of LW radiation emanating from the protostar. We use the same scheme as described in Stacy et al. (2012). Briefly, our raytracing module generates a spherical grid, consisting of $\sim 10^{5}$ rays and 200 radial bins, centered around the first sink. The minimum radius is set equal to the distance between the sink and the nearest neighboring SPH particle, and the grid is updated 
each time ray tracing is performed. Most particles within $r_{\text {acc }}$ from the sink are accreted, so the minimum radius is usually close to 1.0 AU. The bins are logarithmically spaced from the minimum distance to $3 \mathrm{pc}$, the size of the cut-out region. Each SPH particle within a bin then contributes its density and chemical abundances, proportional to its density squared, to the average values assigned to the bin.

Also as in Stacy et al. (2012), we then use the ray-tracing scheme to determine the $\mathrm{H}_{2}$ column density $N_{\mathrm{H}_{2}}$, and from this we determine the shielding factor $f_{\text {shield }}$ with the fitting formula from Draine \& Bertoldi (1996). We note a recent update to the $f_{\text {shield }}$ prescription by Wolcott-Green et al. (2011) and Wolcott-Green \& Haiman (2011), but do not expect this to significantly affect the results for our particular case. We combine this with a protostellar evolution model (see Section 3 ) in which we assume a blackbody spectrum with an effective temperature $T_{\text {eff }}$, as specified by that model. We then determine the approximate LW radiation flux $F_{\mathrm{LW}}$, in units of erg s $\mathrm{s}^{-1} \mathrm{~cm}^{-2} \mathrm{~Hz}^{-1}$, at $h \bar{v}=12.87 \mathrm{eV}$ (Abel et al. 1997). This finally allows for a determination of the $\mathrm{H}_{2}$ dissociation rate,

$$
k_{\mathrm{H}_{2}}=1.1 \times 10^{8} f_{\text {shield }} F_{\mathrm{LW} \mathrm{s}}{ }^{-1},
$$

to be included in our chemical network.

\section{PROTOSTELLAR EVOLUTION MODEL}

\subsection{Luminosity and Temperature Evolution}

Our ray-tracing algorithm requires an input of protostellar effective temperature $T_{\text {eff }}$ and luminosity $L_{*}$. We calculate $L_{*}$ as the sum of $L_{\text {acc }}$, the accretion luminosity, and $L_{\text {int }}$, the luminosity originating from the stellar interior and finally emitted from the photosphere of the protostar. We write $L_{*}$ as

$$
L_{*}=L_{\mathrm{acc}}+L_{\mathrm{int}}=\alpha \frac{G M_{*} \dot{M}}{R_{*}}+L_{\mathrm{int}},
$$

where $M_{*}$ is the protostellar mass, $\dot{M}$ the accretion rate, and $R_{*}$ the protostellar radius (cf. Prialnik \& Livio 1985; Hartmann et al. 1997). We take $M_{*}$ to be the mass of the sink, and $\dot{M}$ to be the accretion rate onto the sink, measured by averaging the total mass growth of the sink over the previous $10 \mathrm{yr}$. If the measured sink accretion rate yields $\dot{M} \simeq 0$, we simply assume the protostar is described by $L_{*}=L_{\text {int }}$.

We define $\alpha$ as the fraction of thermal energy from accretion that is added to the stellar interior. Cold disk accretion would thus be described by $\alpha=0$, while for hot spherical accretion $\alpha=1$. For the main sink particle, we determine $\alpha$ by measuring the $j_{\mathrm{SPH}}$ of each particle accreted by the sink within the last $10 \mathrm{yr}$, as well as each particle currently within $10 \mathrm{AU}$ from the sink. This allows us to find the percentage of nearby and recently accreted particles that have low angular momentum $\left(j_{\mathrm{SPH}}<0.5 j_{\text {cent }}\right)$ versus high angular momentum $\left(j_{\mathrm{SPH}}>\right.$ $\left.0.5 j_{\text {cent }}\right)$. As described in Section $2.5, j_{\mathrm{SPH}}=v_{\text {rot }} d$ is the angular momentum of the gas particle and $j_{\text {cent }}=\sqrt{G M_{\text {sink }} r_{\text {acc }}}$ is the angular momentum required for centrifugal support against infall onto the sink. We take $\alpha$ to be the fraction of particles with $j_{\text {SPH }}<0.5 j_{\text {cent }}$, such that $\alpha=1$ if the near-sink gas is dominated by radial instead of rotational motion.

$L_{\text {int }}$ will vary with the mass of the protostar. When the protostar first forms and has low mass, we assume it is on the Hayashi track of the Hertzsprung-Russell diagram. We approximate this by holding the protostar at an effective temperature of $T_{\text {Hay }}=4500 \mathrm{~K}$ while its luminosity may vary. This yields a "Hayashi track" luminosity of

$$
L_{\text {Hay }}=4 \pi R_{*}^{2} \sigma_{\mathrm{SB}} T_{\text {Hay }}^{4} .
$$

The precise value of $T_{\text {Hay }}$ will vary from $\sim 3000$ to $5000 \mathrm{~K}$ depending upon stellar mass and opacity. Because the protostar we consider is metal-free, the opacity of the protostellar atmosphere will differ from that of a Pop I or Pop II star, and the resulting $T_{\text {Hay }}$ will tend to be marginally higher for Pop III stars (e.g., Stahler et al. 1986a). We thus choose $T_{\text {Hay }}$ to be in the upper end of this range and set it to $4500 \mathrm{~K}$. The uncertain value of initial $T_{\text {eff }}$ (from 3000 to $5000 \mathrm{~K}$ ) corresponds to a $L_{\text {Hay }}$ that may vary by a factor of eight. However, a $T_{\text {eff }}$ even in the upper end of this range will not lead to significant feedback until after the protostar leaves the Hayashi track. Our simulation results are thus not sensitive to the choice of initial $T_{\text {eff }}$.

If the protostar grows sufficiently massive, we assume it eventually transitions to the Henyey track (Henyey et al. 1955, see also Hansen et al. 2004), and will gradually contract down to the main-sequence radius and commence hydrogen burning. However, the protostellar system in our simulation exhibits unusually low mass, and thus has much longer evolutionary timescales than the more common high-mass Pop III stellar systems. We thus do not follow sufficiently long timescales for the protostars to reach these later stages, so we do not discuss them here. Our protostellar model at these early times predicts a typical $L_{*}$ and $T_{\text {eff }}$ of $\sim 100 L_{\odot}$ and $4500 \mathrm{~K}$. This luminosity can roughly double during periods of rapid accretion due to the contribution from $L_{\text {acc }}$, while the corresponding $T_{\text {eff }}$ will increase by a few hundred kelvins. At such low $T_{\text {eff }}$, the fraction of luminosity in the LW band is negligible. Combined with significant $\mathrm{H}_{2}$ shielding in the disk, LW dissociation is unimportant at these early times.

\subsection{Radial Evolution}

As discussed in previous one-dimensional studies (Stahler et al. 1986b; Omukai \& Palla 2003; Hosokawa et al. 2010), a growing protostar initially undergoes an "adiabatic accretion" phase, where $R_{*}$ grows with mass. This continues approximately while $t_{\mathrm{acc}}<t_{\mathrm{KH}}$, where

$$
t_{\mathrm{KH}}=\frac{G M_{*}^{2}}{R_{*} L_{*}}
$$

is the Kelvin-Helmholtz (KH) timescale and

$$
t_{\mathrm{acc}}=\frac{M_{*}}{\dot{M}}
$$

is the accretion timescale. The protostar will later begin $\mathrm{KH}$ contraction approximately when $t_{\mathrm{acc}}>t_{\mathrm{KH}}$.

Hosokawa et al. (2010) modeled the evolution of a primordial protostars growing at $\dot{M}=10^{-3} M_{\odot} \mathrm{yr}^{-1}$. They found that, particularly during the "adiabatic" phase, accretion through a geometrically thin disk will lead to smaller protostellar radii than spherically symmetric accretion. The true accretion geometry is likely somewhere in between the idealized "disk" and "spherical" cases, with the infall beginning as nearly spherical and growing gradually more disk-like over time. Given the spherical accretion case described in Omukai \& Palla (2003; see also Stahler et al. 1986b), the radial evolution during the adiabatic accretion phase can be described by the following 
expression:

$$
R_{I, \text { sphere }} \simeq 49 R_{\odot}\left(\frac{M_{*}}{M_{\odot}}\right)^{1 / 3}\left(\frac{\dot{M}}{\dot{M}_{\mathrm{fid}}}\right)^{1 / 3} .
$$

where $\dot{M}_{\text {fid }} \simeq 4.4 \times 10^{-3} M_{\odot} \mathrm{yr}^{-1}$, the fiducial accretion rate used in the abovementioned studies (see also Stacy et al. 2010).

If a protostar transitions from spherical to disk accretion, the radial evolution during the "adiabatic accretion" phase will be significantly different from the purely spherical case. For the range of accretion rates studied in Hosokawa et al. (2010), after transitioning to disk accretion, the radius rapidly declines due to the decrease in entropy brought to the stellar interior. For pure disk accretion, this decline can be described by

$$
R_{I, \text { disk }} \simeq R_{0}\left(\frac{M_{*}}{M_{0}}\right)^{-0.63},
$$

where $R_{0}$ and $M_{0}$ are the protostellar mass and radius at the point of transition from spherical to disk accretion. In our case, the protostar's accretion is disky even from initial sink formation, so we simply use the initial sink mass to set $M_{0}=0.045 M_{\odot}$. Following the disk accretion model of Hosokawa et al. (2010; their Figure 4), we approximate $R_{0}=1.7 M_{*}^{(-1 / 3)}=5 R_{\odot}$.

We subsequently set our radius in between the spherical and disk cases such that

$$
R_{I}=\alpha R_{I, \text { sphere }}+(1-\alpha) R_{I, \text { disk }}
$$

If the radial decline is unphysically rapid $\left(\dot{R}<-R / t_{\mathrm{KH}}\right)$, we limit the rate of contraction to be $\dot{R}=-R / t_{\mathrm{KH}}$. The radial decline will continue until deuterium burning begins in the stellar interior, thus increasing the average entropy within the star, which occurs when $T_{\text {int }}>2 \times 10^{6} \mathrm{~K}$. After this point the protostar again undergoes a roughly adiabatic expansion. When a maximum radius is reached (see Hosokawa et al. 2010), $\mathrm{KH}$ contraction to the main sequence will begin. However, our simulations do not follow the protostellar growth to these later stages because we are examining an atypical low-mass case in which the protostellar evolutionary timescales are very long compared to those of more massive protostars.

In our model $R_{*}$ expands to nearly $30 R_{\odot}$ over the first few hundred years during a period of more spherical-type accretion. After a transition to more disk-type accretion, $R_{*}$ gradually declines as $\dot{R}=-R / t_{\mathrm{KH}}$ down to $\sim 15 R_{\odot}$ by the end of the simulation.

\section{RESULTS}

\subsection{Initial Minihalo Collapse}

The chemical and thermal evolution of the central minihalo gas up to the formation of the first sink particle is depicted by the red lines in Figure 1. The minihalo is in place by $z=15$, and the subsequent evolution is similar to that of previous work (e.g., Yoshida et al. 2006; Greif et al. 2011). The gas is heated through adiabatic compression as it approaches densities of $10^{8} \mathrm{~cm}^{-3}$. After this point, three-body reactions rapidly increase the $\mathrm{H}_{2}$ fraction, such that the correspondingly enhanced cooling rate is similar to the combination of the $\mathrm{H}_{2}$ formation heating rate and adiabatic heating rate due to collapse, yielding a roughly isothermal evolution. The gas becomes fully molecular by densities of $10^{10} \mathrm{~cm}^{-3}$, forming a $\sim 1 M_{\odot}$ molecular core. Above these densities the $\mathrm{H}_{2}$ cooling is no longer optically thin and the gas gradually heats again to $\sim 2000 \mathrm{~K}$ by $n=10^{16} \mathrm{~cm}^{-3}$, at which point the first sink particle forms.

The velocity structure of the central 10,000 AU is shown in Figure 2. Within each logarithmically spaced radial bin, we take $v_{\text {rad }}$ and $v_{\text {rot }}$ as the mass-weighted average of the individual particle velocities within each bin. Both the radial and rotational gas velocity $v_{\text {rad }}$ and $v_{\text {rot }}$ are on the order of half of $v_{\mathrm{ff}}$, where $v_{\mathrm{ff}}$ is the free-fall velocity based upon the enclosed mass at the given radius. The gas has a substantial amount of rotational support such that $v_{\text {rot }}$ dominates over $v_{\text {rad }}$ and is approximately half of the Keplerian velocity $v_{\text {Kep }}$.

In a similar fashion, we measure the turbulent Mach number, $M_{\text {turb }}$, over the same range of radial bins according to

$$
M_{\mathrm{turb}}^{2} c_{s}^{2}=\sum_{i} \frac{m_{i}}{M}\left(\boldsymbol{v}_{i}-\boldsymbol{v}_{\mathrm{rot}}-\boldsymbol{v}_{\mathrm{rad}}\right)^{2},
$$

where $c_{s}$ is the sound speed of the radial bin, $m_{i}$ is the mass of a gas particle contributing to the bin, and $M$ is the total gas mass of the bin. The central 10,000 AU of gas are characterized by subsonic and nearly sonic turbulence.

\subsection{Comparison with Other Minihalos}

\subsubsection{Global Minihalo Characteristics}

As will be discussed in Section 4.3, our simulated Pop III system has an unusually low accretion rate. We here examine whether this is due to the characteristics of its host minihalo. We first measure the evolution of the virial mass of the minihalo considered here, and compare to four other minihalos taken from the cosmological simulations presented in Greif et al. (2012) and Stacy \& Bromm (2013), where we have chosen the minihalos hosting the most rapidly and most slowly accreting stellar systems from each of those two studies. We determine which particles reside in the halo by first locating the simulation region's densest gas particle, or hydrodynamic mesh element in the case of the AREPO simulations. Making the simple assumption that this point marks the center of the halo, the extent of the halo was determined by finding the surrounding spherical region in which the average DM density is $200 \rho_{b}$, where $\rho_{b} \simeq 2.5 \times 10^{-30}(1+z)^{3} \mathrm{~g} \mathrm{~cm}^{-3}$ is the redshiftdependent background density.

The minihalo of our simulation has the minimum necessary mass of $M_{\text {halo }} \lesssim 10^{6} M_{\odot}$ before gas condensation and $\mathrm{H}_{2}$ cooling begins, and this is very close to the mass of other Pop III star-forming halos (Figure 3). However, it does not reach this minimum $M_{\text {halo }}$ until the relatively late time of $z=15$. By this redshift, Hubble expansion has reduced the density of the background universe, leading to a slower accretion rate from the surrounding cosmic web. In particular, the average DM accretion rate $\dot{M}_{\mathrm{DM}}$ of our minihalo is $3 \times 10^{-3} M_{\odot} \mathrm{yr}^{-1}$, as compared to the more typical rate of $10^{-2} M_{\odot} \mathrm{yr}^{-1}$ for minihalos which collapsed at $z \sim 30$. Employing this same redshift range and $M_{\mathrm{DM}}=5 \times 10^{5} M_{\odot}$, we find that our measured $\dot{M}_{\mathrm{DM}}$ values are in excellent agreement with analytical fits determined from other numerical simulations, e.g.,

$$
\dot{M}_{\mathrm{DM}} \simeq 35(1+z)^{2.2} M_{12}^{1.07} M_{\odot} \mathrm{yr}^{-1}
$$

where $M_{12}=M_{\mathrm{DM}} / 10^{12} M_{\odot}$ (Genel et al. 2008). Though the above estimate was originally derived from simulations of much larger-mass halos, it also applies accurately to our minihalos. Genel et al. (2008) furthermore find that the above 

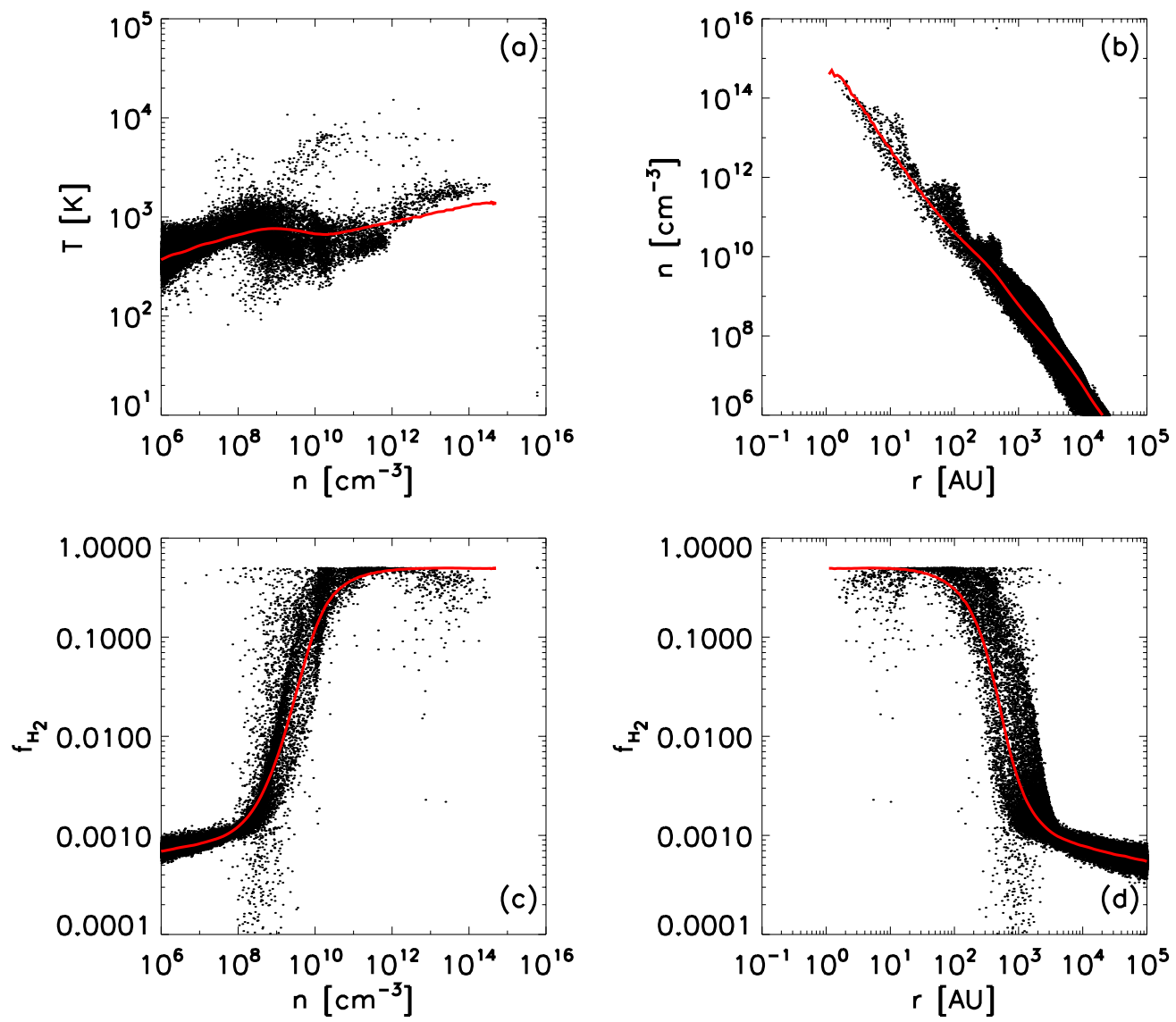

Figure 1. Physical state of the minihalo gas $3000 \mathrm{yr}$ after the formation of the first sink particle. (a) Temperature vs. number density $n$. (b) $n$ vs. distance $r$ from the main sink particle. (c) $\mathrm{H}_{2}$ fraction $f_{\mathrm{H}_{2}}$ vs. $n$. (d) $\mathrm{H}_{2}$ fraction vs. distance $r$. Red line shows the radially averaged values of the same quantities just prior to the formation of the first sink, as measured from the densest gas particle. Note the warm phase of gas at $n \gtrsim 10^{7} \mathrm{~cm}^{-3}$, where the gas has been warmed through gravitational heating provided by the main sink.

(A color version of this figure is available in the online journal.)

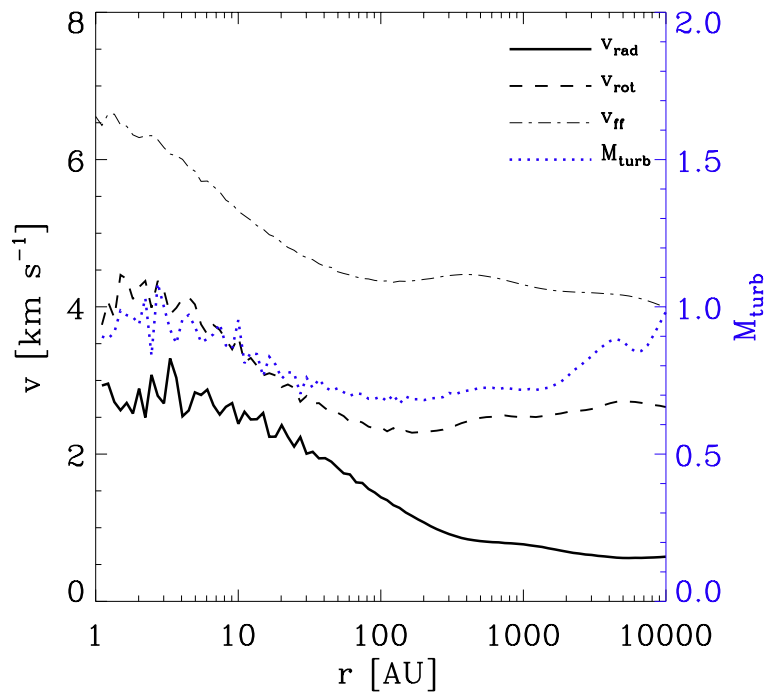

Figure 2. Velocity profile of the gas just prior to initial sink formation. Solid line is the magnitude of the radial infall velocity $v_{\text {rad }}$, averaged within a range of logarithmic radial bins. Dashed line is the average rotational velocity $v_{\text {rot }}$ within these same bins. Dash-dot line is the free-fall velocity $v_{\mathrm{ff}}$ based upon the enclosed mass at each radius. Dotted blue line is the turbulent Mach number $M_{\text {turb }}$, which corresponds to the right-hand $y$-axis scale. The values of $v_{\text {rad }}$ are low compared to $v_{\mathrm{ff}}$, and the magnitude of $v_{\text {rot }}$ dominates over $v_{\text {rad }}$ over all distances shown.

(A color version of this figure is available in the online journal.) expression agrees well with the general predictions of extended Press-Schechter (EPS) theory (Press \& Schechter 1974; Mo \& White 1996; Lacey \& Cole 1993, see also Neistein et al. 2006). As numerically confirmed in Gao et al. (2005; e.g., their Figure 1), the Press-Schechter formalism indeed accurately predicts the accretion rate of minihalos with $M_{\text {halo }} \sim 10^{6} M_{\odot}$. They found an accretion rate of $\sim 2.5 \times 10^{-2} M_{\odot} \mathrm{yr}^{-1}$ for their $z=50$ minihalo when it was collecting the bulk of its mass, which in turn corresponds well with the above equation's prediction of $\sim 2 \times 10^{-2} M_{\odot} \mathrm{yr}^{-1}$. This further confirms the expected larger growth rates of these rare-peak and high-redshift minihalos (e.g., Reed et al. 2005).

The gas mass within the minihalo, which we take simply as all gas within the halo virial radius with $n>1 \mathrm{~cm}^{-3}$, grows at average rates which range from $M_{\text {gas }}=10^{-4} M_{\odot} \mathrm{yr}^{-1}$ for our minihalo to $10^{-3} M_{\odot} \mathrm{yr}^{-1}$ for the earlier-collapsing minihalos. This may be compared to the growth rate found in previous studies, e.g.,

$$
\dot{M}_{\text {gas }} \simeq 6.6(1+z)^{2.25} M_{12}^{1.15} f_{0.165} M_{\odot} \mathrm{yr}^{-1},
$$

where $f_{0.165}$ is the baryonic fraction in the halos in units of the cosmological value $f_{\mathrm{B}}=\Omega_{\mathrm{B}} / \Omega_{\mathrm{M}}=0.165$ (e.g., Dekel et al. 2009; see also Faucher-Giguère et al. 2011). Assuming $f_{0.165}=1$ we find that, over the redshift range $z=15-30, \dot{M}_{\text {gas }}$ ranges from 2 to $7 \times 10^{-4} M_{\odot} \mathrm{yr}^{-1}$. Thus, for a given DM halo mass (e.g., $M_{\text {halo }}=10^{6} M_{\odot}$ ), the rate at which both DM and gas 

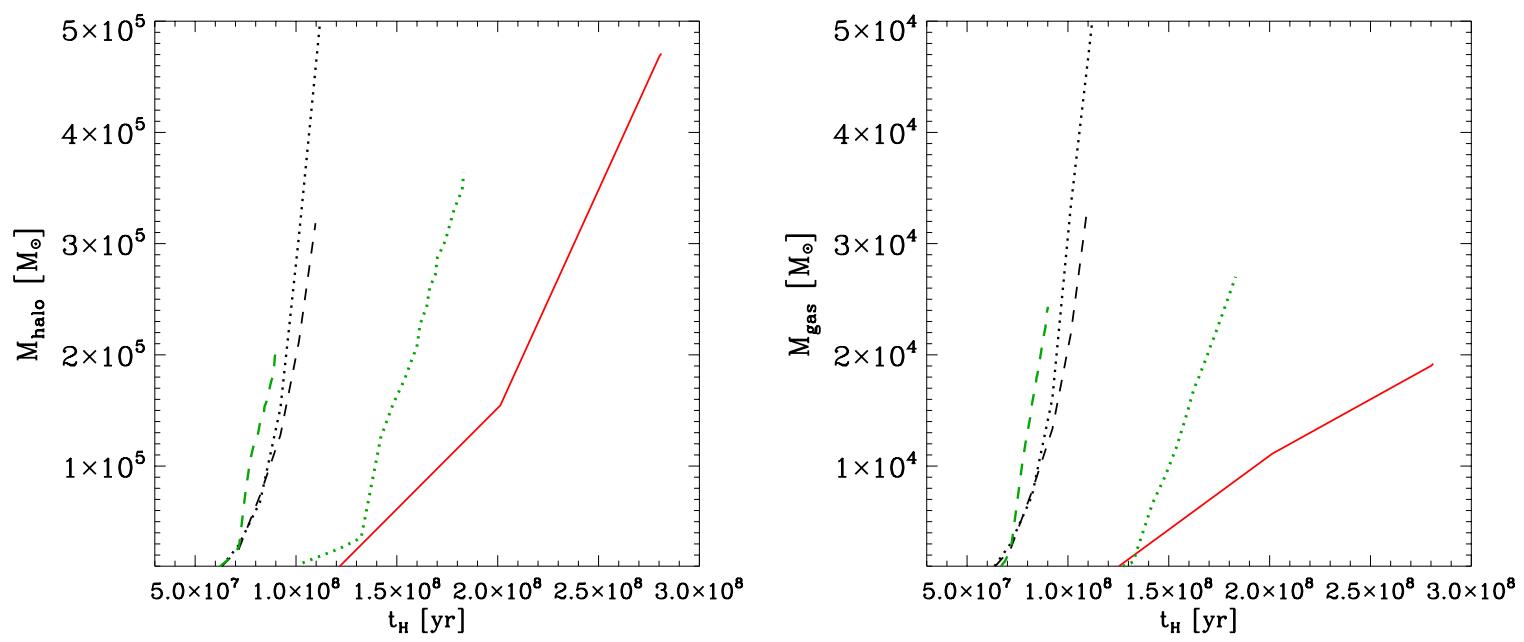

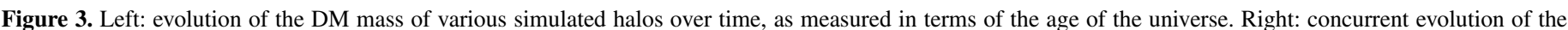

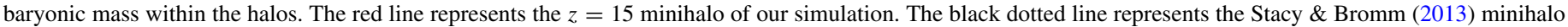

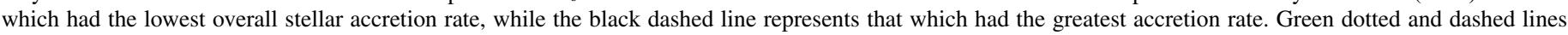
additionally show the growth of the two minihalos from Greif et al. (2012) which had the lowest and highest accretion rates, respectively.

(A color version of this figure is available in the online journal.)

will fall into the gravitational potential well will vary by nearly an order of magnitude over this redshift range. This corresponds to the nearly factor of ten reduction in $\rho_{b}$ as the universe expands over redshifts $z=30$ to $z=15$.

The variation in collapse redshifts seen in these simulations stems from differences in sizes of the cosmological box that was used. The Greif et al. (2012) and Stacy \& Bromm (2013) simulations employed box sizes of $500 \mathrm{kpc}$ and $1.4 \mathrm{Mpc}$ (comoving), and were thus able to capture larger " $v \sigma$ peak" fluctuations, where $\sigma$ is the standard deviation in the Gaussian random field of primordial density fluctuations. $\Lambda \mathrm{CDM}$ theory predicts that at $z \sim 30$, a $10^{6} M_{\odot}$ halo corresponds approximately to a $3 \sigma$ peak (e.g., Loeb 2010; Bromm 2013). Our smaller box size of $200 \mathrm{kpc}$ (comoving) captures only $1 \sigma-2 \sigma$ peaks, which instead corresponds to a later collapse redshift of $z \sim 15$ for a $10^{6} M_{\odot}$ halo.

\subsubsection{Star-forming Core Characteristics}

Along with slower overall halo gas accretion in the $z=$ 15 minihalo, we find a reduced gas accretion rate within the central parts of the minihalo as well, which may be roughly estimated through the gas soundspeed $c_{\mathrm{s}}$ (Figure 4). Although we find good agreement in $c_{\mathrm{s}}$ beyond $10^{5} \mathrm{AU}(\sim 0.5 \mathrm{pc})$, there is divergence in $c_{\mathrm{s}}$ in the inner regions. In our simulation the sound speed of the gas within $10^{4} \mathrm{AU}$ is $\sim 3 \mathrm{~km} \mathrm{~s}^{-1}$. This is slightly less than that seen in some of the comparison halos, $\sim 4 \mathrm{~km} \mathrm{~s}^{-1}$ (Figure 4). Making the simple estimate that the Jeans mass is infalling at the free-fall rate, we can scale the accretion rate with soundspeed as $\dot{M} \simeq c_{\mathrm{s}}^{3} / G$, similar to the Shu (1977) similarity solution for collapse of a singular isothermal sphere. From this we may predict accretion rates ranging from $\sim 6 \times 10^{-3} M_{\odot} \mathrm{yr}^{-1}$ for the $z=15$ minihalo to $\sim 1.5 \times 10^{-2} M_{\odot} \mathrm{yr}^{-1}$ for the higherredshift halos.

Other properties of the gas, such as the density profile and $\mathrm{H}_{2}$ fraction (lower panels of Figure 4), show interesting variation between minihalos, as well. At distances greater than $100 \mathrm{AU}$, the $\mathrm{H}_{2}$ fraction can vary by approximately an order of magnitude, though in each star-forming cloud the gas is fully molecular within the central $100 \mathrm{AU}$. We do not find an exact correlation between $\mathrm{H}_{2}$ fraction and $c_{\mathrm{s}}$. However, our $z=15$ minihalo generally has the lowest $\mathrm{H}_{2}$ fraction at distances greater than $1000 \mathrm{AU}$ as well as nearly the lowest temperatures at all distances. The gas density is unusually small as well. Though for each star-forming core the density roughly follows a $\rho \propto r^{-2}$ profile, beyond 20 AU the $z=15$ minihalo profile is normalized to smaller values than the others. At some radii the gas density is an order of magnitude lower than the minihalo with the highest density.

As shown in Figures 4 and 5, we may also approximate the spherical accretion rate $\dot{M}_{\text {sph }}$ that results from the density and radial velocity profiles, which is appropriate when considering gas within the minihalo where the density profile is more spherically symmetric. We estimate $\dot{M}_{\text {sph }}$ within a range of radial bins as

$$
\dot{M}_{\text {sph }}=4 \pi r^{2} \rho v_{\text {rad }},
$$

where $r$ is the distance as measured from the densest gas particle, and $v_{\text {rad }}$ is the average radial velocity of gas within the radial bin. As is apparent in Figure 4, between $100 \mathrm{AU}$ and $10^{5} \mathrm{AU}(0.5 \mathrm{pc})$ the gas within our minihalo typically infalls at $v_{\text {rad }} \sim 1 \mathrm{~km} \mathrm{~s}^{-1}$, several times smaller than $v_{\text {rad }} \sim 4 \mathrm{~km} \mathrm{~s}^{-1}$ as seen in the most rapidly growing halos. Together with the gas density this yields a gas accretion rate between 100 and $10^{5} \mathrm{AU}$ that ranges from $\sim 6 \times 10^{-4} M_{\odot} \mathrm{yr}^{-1}$ to $\sim 3 \times 10^{-3} M_{\odot} \mathrm{yr}^{-1}$, approximately an order of magnitude less than the accretion rates found in the high-redshift halos. For all minihalos, $\dot{M}_{\text {sph }}$ is also substantially lower than the accretion rate estimated from $c_{\mathrm{s}}$, indicating that angular momentum support slows gas infall. We furthermore note that the halo which formed at the second-most recent redshift (green dotted lines in Figures 3-5) has DM and gas accretion rates and $\dot{M}_{\text {sph }}$ values which are intermediate between the $z=15$ minihalo and the highest-redshift halos.

These results may indicate a correlation between collapse redshift and rate of mass infall even on small scales. However, Gao et al. (2007) found that while gas within higher-redshift halos will indeed reach protostellar densities in shorter timescales, there was no subsequent correlation between formation redshift and instantaneous accretion rate at the densities they resolved $\left(10^{10} \mathrm{~cm}^{-3}\right)$. O'Shea \& Norman (2007) even find that Pop III star-forming regions which form later have higher maximum accretion rates, the opposite trend to ours. They attribute 

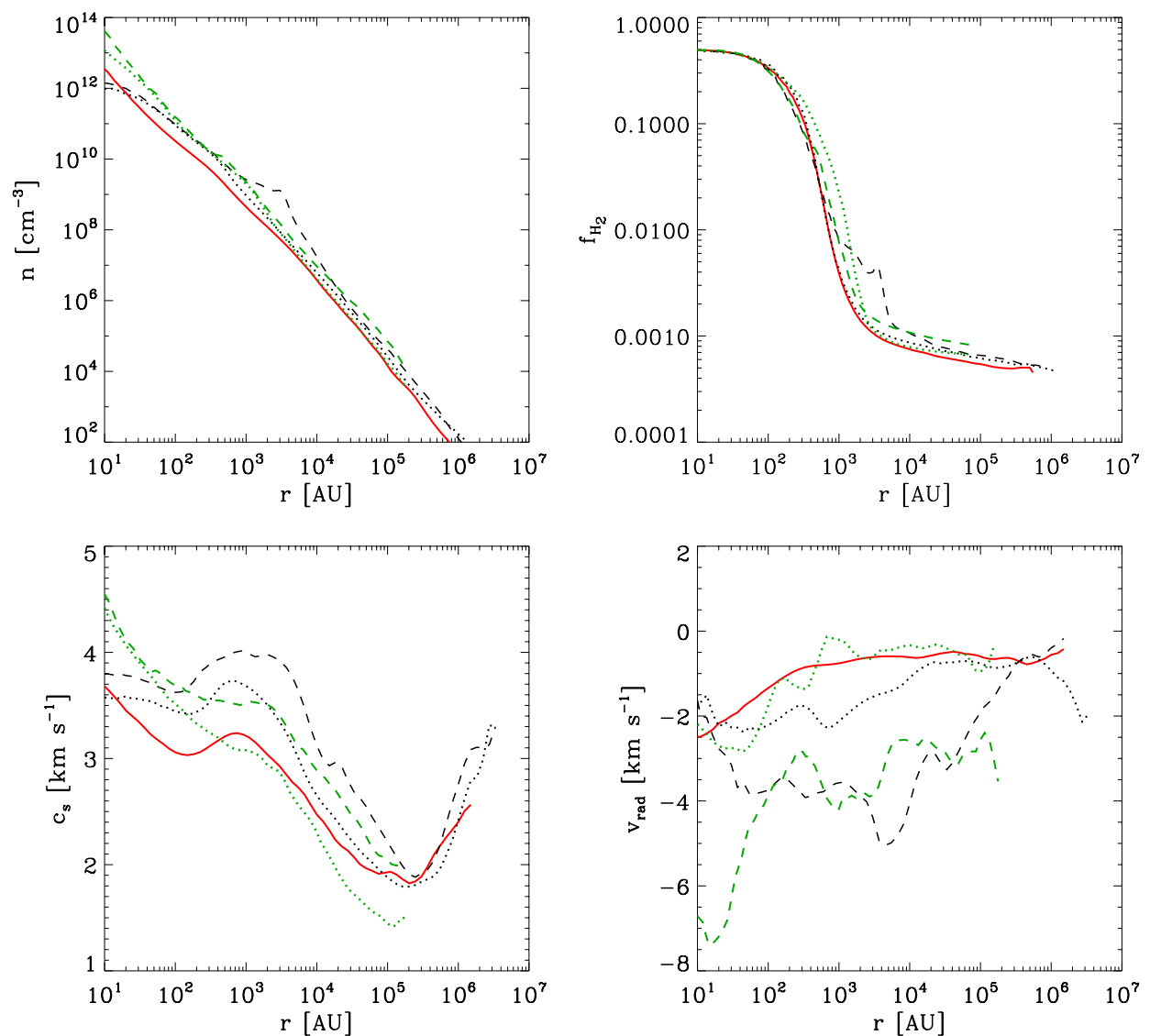

Figure 4. Radial profiles of various gas properties with respect to the highest-density particle, just prior to the initial protostar or sink particle formation. In all panels, dashed and dotted lines represent minihalos from simulations presented in Stacy \& Bromm (2013) and Greif et al. (2012), as described in the previous figure. Upper left: number density vs. radius. Upper right: $\mathrm{H}_{2}$ fraction vs. radius. Lower left: profile of sound speed $c_{\mathrm{s}}$. Lower right: profile of $v_{\text {rad. The combination of smaller }}$ sound speed, as well as lower $\left|v_{\text {rad }}\right|$ and number density, lead to unusually low accretion rates within our $z=15$ minihalo as compared with those from other studies.

(A color version of this figure is available in the online journal.)

this to the increasing virial temperature of halos with redshift, $T_{\mathrm{vir}} \propto M_{\mathrm{vir}}^{2 / 3}(1+z)$. In their chain of reasoning, this would lead to warmer overall gas temperatures as the minihalo first collapses, yielding more rapid $\mathrm{H}_{2}$ formation rates. The higher $\mathrm{H}_{2}$ fraction in turn would lead to cooler gas in the cores of the halos, and thereby slower accretion rates. In Figure 4, however, we find a counterexample to this. A relatively high-redshift minihalo with an enhanced $\mathrm{H}_{2}$ fraction (black dashed line) in fact has the warmest temperatures in both the core and outer region, and in some regions beyond $100 \mathrm{AU}$, it also has the highest accretion rates.

Gao et al. (2007), on the other hand, emphasize the importance of angular momentum. They find that as gas collapses its properties become independent of the global properties of the halo, and that more disk-like and rotationally supported inner star-forming clouds will have lower accretion rates. In the bottom panel of Figure 5 we compare the levels of rotational support throughout the halos, defined as $f_{\text {rot }}=v_{\text {rot }} / v_{\text {Kep }}$, where $v_{\text {Kep }}=\left(G M_{\text {enc }} / r\right)^{1 / 2}$. The $z=15$ minihalo has $f_{\text {rot }} \sim 0.6$ over several orders of magnitude in distance. The other two more slowly accreting systems (green and black dotted lines in Figure 5) also maintain high $f_{\text {rot }}$ between 10 and $10^{6} \mathrm{AU}$, with $f_{\text {rot }}$ consistently greater than 0.6 . In contrast, the two most rapidly accreting systems (green and black dashed lines) have extended regions where $f_{\text {rot }}$ falls below 0.5. Thus, similar to the conclusions of Gao et al. (2007), angular momentum structure, combined with that of temperature and density, plays a key role in the rate of gas infall.
Despite variation in the level of rotational support, the specific angular momentum profile of the central gas for each minihalo we examine, shown in Figure 6, all follow a similar $j_{\text {tot }} \propto M_{\text {enc }}$ power law. However, it is interesting to note that the profiles of the two most rapidly accreting halos (green and black dashed lines in Figure 6) are normalized at nearly a factor of two lower than the minihalo of our simulation. The greater rates at which the central gas flows inward (Figure 4), as well as the reduced rotational support (Figure 5, bottom panel), indicates that in these halos $v_{\text {rad }}$ dominates over $v_{\text {rot }}$, leading to higher accretion rates at the point of protostellar formation.

As expected, the mass enclosed within a given radius is also larger for those minihalos with less rotational support. The resulting ratio of enclosed mass $M_{\text {enc }}$ to Bonnor-Ebert mass $M_{\mathrm{BE}}$ is larger as well (Figure 7). We estimate $M_{\mathrm{BE}}$ as

$$
M_{\mathrm{BE}} \simeq 1000 M_{\odot}\left(\frac{T}{200 \mathrm{~K}}\right)^{3 / 2}\left(\frac{n}{10^{4} \mathrm{~cm}^{-3}}\right)^{-1 / 2}
$$

$M_{\mathrm{BE}}$ is similar to the Jeans mass, and a ratio of $M_{\mathrm{enc}}$ to $M_{\mathrm{BE}}$ that is close to unity roughly indicates gravitational instability of the gas.

We note that de Souza et al. (2013) also emphasize the influence of rotation on the Pop III IMF, finding from their cosmological simulation that the spin distribution of gas within minihalos evolves with redshift. They employed the model from McKee \& Tan (2008) which used semi-analytic methods to find a relation between gas rotational support, effectiveness of 

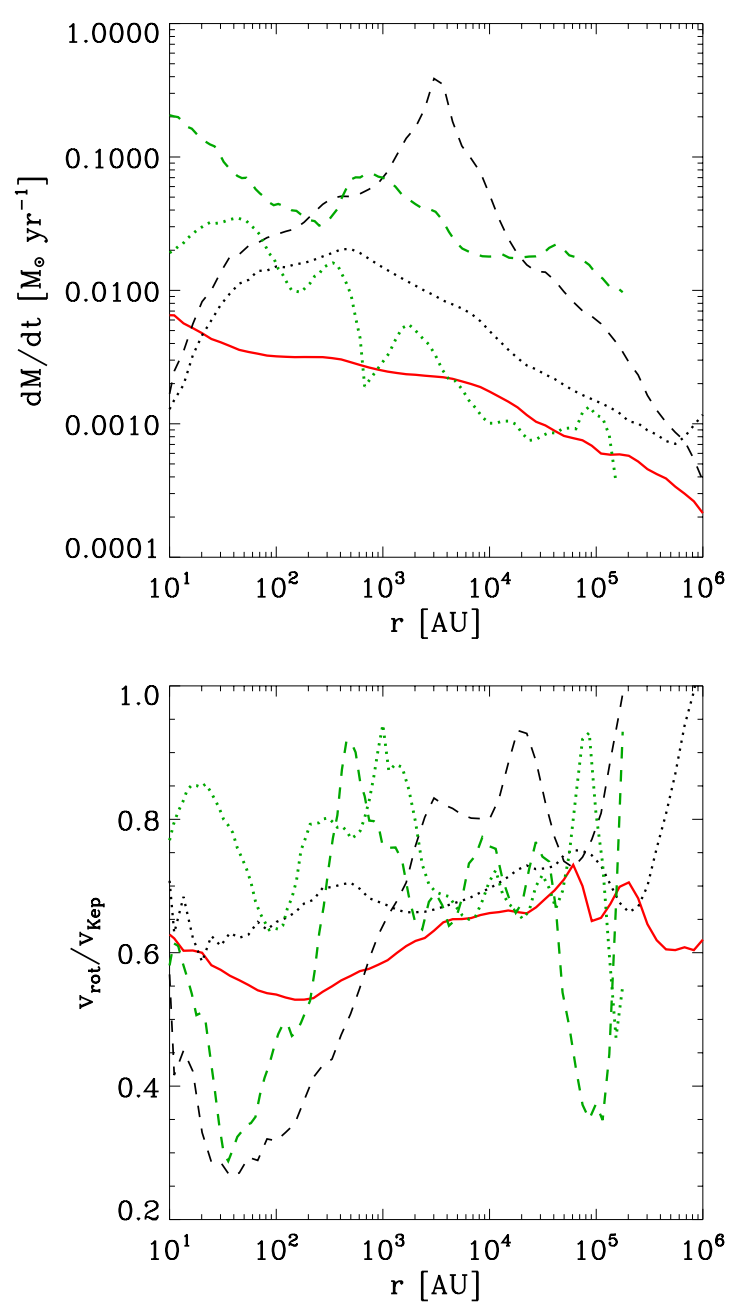

Figure 5. Top: estimated spherical accretion rate $\dot{M}_{\text {sph }}$ over a range of distances from densest gas particle, just prior to protostar or sink particle formation. Line styles have the same meaning as in previous figures. Note that the combination of reduced density and $\left|v_{\text {rad }}\right|$ within the inner few $10^{5} \mathrm{AU}(\sim 0.5 \mathrm{pc})$ leads to lower accretion rates than typically found in primordial gas. Bottom: level of rotational support $f_{\text {rot }}=v_{\text {rot }} / v_{\text {Kep }}$ throughout the gas at this same time. Note that the two most rapidly accreting systems (green and black dashed lines) are also the only two systems with extended regions of very low rotational support $\left(f_{\text {rot }}<0.5\right)$.

(A color version of this figure is available in the online journal.)

protostellar feedback, and ultimate protostellar mass. Assuming one star per minihalo, de Souza et al. (2013) determine that the Pop III IMF should evolve to have lower peak masses at lower redshift. However, they point out that correlating spin with a Pop III IMF will be complicated by further feedback effects and Pop III multiplicity.

Our results concerning the relation between angular momentum and protostellar accretion rate agree with the those of Gao et al. (2007). The implication that the IMF may shift to lower mass with lower redshift also shows a rough agreement with de Souza et al. (2013), but for different physical reasons. However, neither these authors nor O'Shea \& Norman (2007) simulated the evolving accretion rate of the protostellar cloud after the first protostar formed. Our study allows for this through the sink particle method. As will be further discussed in Section 4.3, we find that the slower and more rotationally dominated gas infall within the $z=15$ minihalo later leads to reduced accretion rates on to the evolving stellar system as well. In Figure 8 we compare the total sink mass after $5000 \mathrm{yr}$ and the total angular momen-

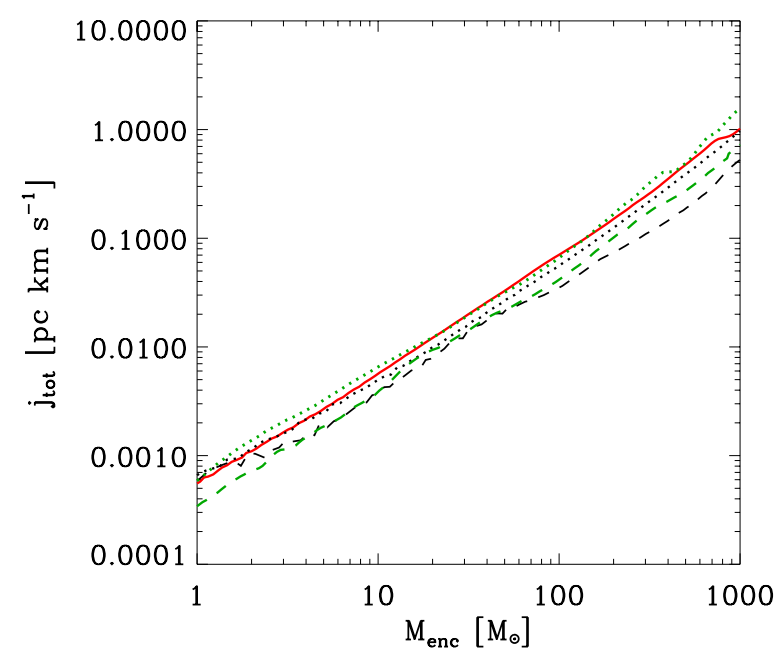

Figure 6. Angular momentum profiles from various simulated minihalos, taken at the point of first sink or protostar formation. The solid red line is taken from the simulation discussed here. The dashed black line is the most rapidly accreting cluster from Stacy \& Bromm (2013). The dotted black line is the most slowly accreting cluster from Stacy \& Bromm (2013). The green dotted and dashed lines are taken from Greif et al. (2012) minihalos which had the lowest and highest accretion rates, respectively.

(A color version of this figure is available in the online journal.)

tum within the central $200 M_{\odot}$ just prior to sink formation. We use the minihalo of this work as well as the suite of ten minihalos presented in Stacy \& Bromm (2013). Some anti-correlation is apparent, particularly in that the $z=15$ minihalo has both the lowest total stellar mass and the highest central angular momentum. However, this trend does have significant scatter since other processes such as turbulent angular momentum transport and $\mathrm{N}$-body dynamics will affect the growth rate of the stellar cluster.

\subsection{Evolution of Protostellar Disk}

As the gas collapses and begins to form sink particles, it also develops into a flattened disk structure (Figure 9). We may estimate the dependence of the mass inflow rate inside the accretion disk on density and temperature using the following:

$$
\dot{M}_{\text {disk }}=3 \pi \nu \Sigma,
$$

where $\Sigma$ is the disk surface density and $v$ is estimated based upon the prescription introduced by Shakura \& Sunyaev (1973),

$$
v=\alpha_{\mathrm{SS}} H_{\mathrm{p}} c_{\mathrm{s}}
$$

$H_{\mathrm{p}}$ is the pressure scale height of the disk and $\alpha_{\mathrm{SS}}$ is a non-dimensional parameter ranging between $\sim 10^{-2}$ and 1 , depending on the nature of angular momentum transport in the disk. In Figure 10 we show the inner surface density profile as measured within a thin $20 \mathrm{AU}$ slice through the central disk, measured just prior to the formation of the first sink particle. We compare with the same set of other minihalos as previously discussed. Note that the length of $20 \mathrm{AU}$ is chosen because this is the resolution limit of the comparison minihalos first presented in Stacy \& Bromm (2013). Even on scales as small as $100 \mathrm{AU}$, the disk has surface densities as much as three times lower than the comparison minihalos. From Equation (16) we see that this leads to a correspondingly reduced disk accretion rate which persists well after the first sink appears.

Let us roughly estimate $\alpha_{\mathrm{SS}} \simeq 0.1$ and $c_{s} \sim 3 \mathrm{~km} \mathrm{~s}^{-1}$, corresponding to a temperature of $\sim 1000 \mathrm{~K}$. The disk scale 

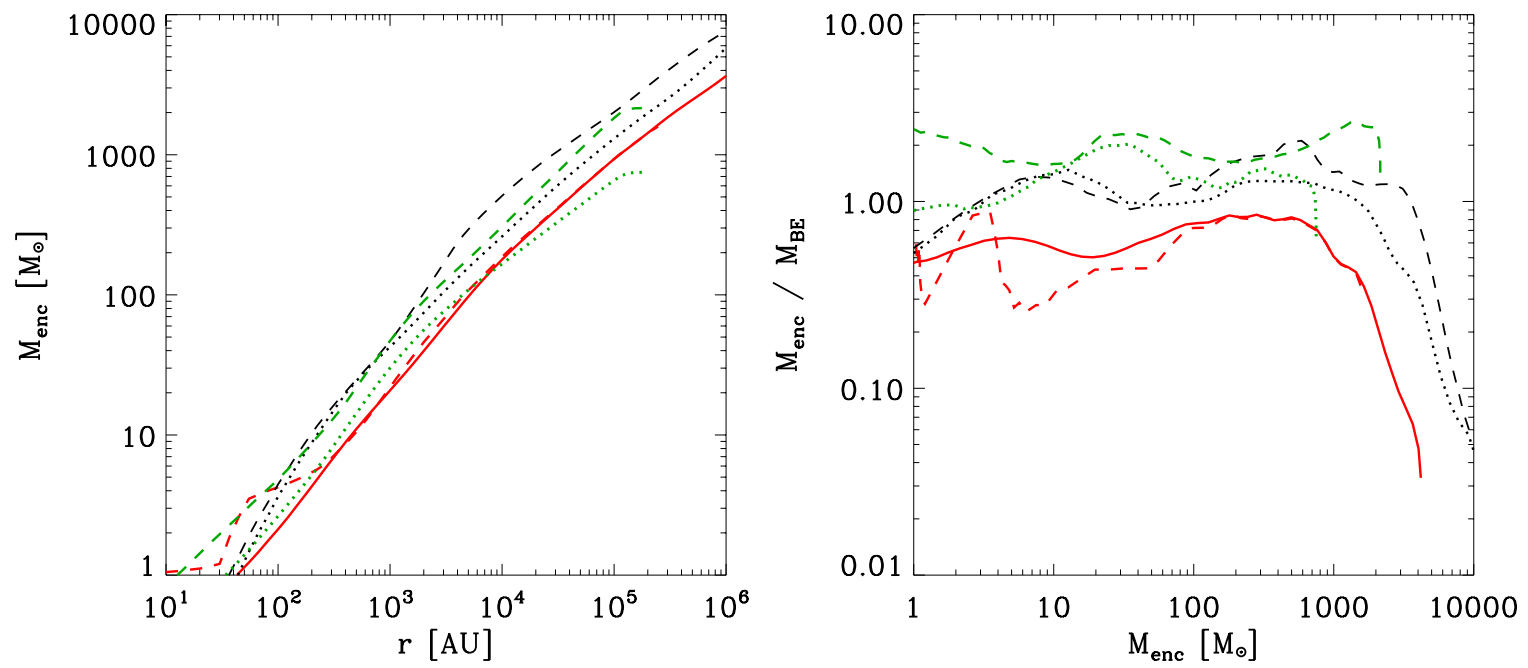

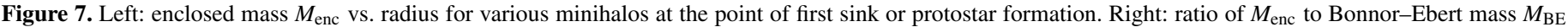

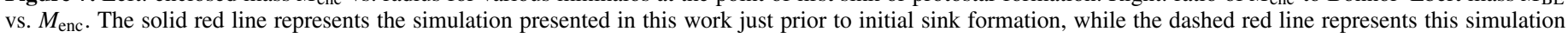

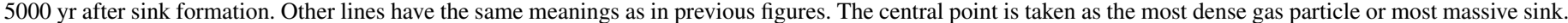
Minihalos with higher overall accretion rates and less rotational support have lower overall $M_{\mathrm{enc}}$ at a given radius and a lower ratio of $M_{\mathrm{enc}}$ to $M_{\mathrm{BE}}$.

(A color version of this figure is available in the online journal.)

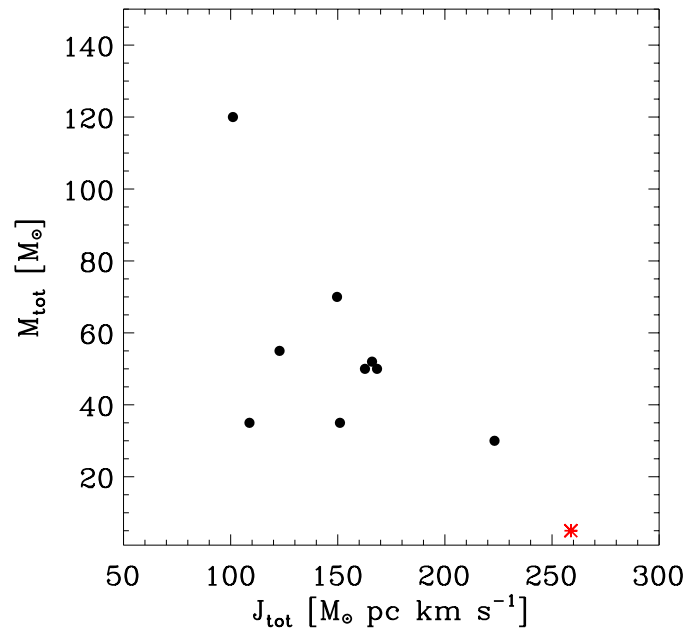

Figure 8. Total mass accreted onto the stellar system after $5000 \mathrm{yr}$ vs. the total angular momentum within the central $200 M_{\odot}$ of enclosed mass. Angular momentum is measured just before the first sink forms. Values are taken from this simulation (red asterisk) as well as the minihalos studied in Stacy \& Bromm (2013; filled circles). Note that the gas with the highest angular momentum also has the lowest stellar mass and accretion rate.

(A color version of this figure is available in the online journal.)

height at $R=100$ AU may be estimated as $H_{\mathrm{p}} / R \sim c_{s} / v_{\text {rot }}(R)$. From Figure 2 we estimate $v_{\text {rot }}(R=100 \mathrm{AU}) \sim 3 \mathrm{~km} \mathrm{~s}^{-1}$ and thus $H_{\mathrm{p}} \sim 100 \mathrm{AU}$. We then find $v \sim 5 \times 10^{19} \mathrm{~cm}^{2} \mathrm{~s}^{-1}$.

The surface density at $100 \mathrm{AU}$ ranges from 30 to $100 \mathrm{~g} \mathrm{~cm}^{-1}$, yielding $\dot{M}_{\text {disk }}$ rates which range from $2 \times 10^{-4}$ to $6 \times$ $10^{-4} M_{\odot} \mathrm{yr}^{-1}$. Assuming the sinks grow at similar rates, we would expect after $10,000 \mathrm{yr}$ that the stellar systems would reach a total mass of 2-6 $M_{\odot}$. The lower end of this mass range is within good agreement with the total mass accretion rate seen in the simulation. However, the upper end is still somewhat lower than the total sink masses found in other calculations (Figure 8). Further variations in $\dot{M}_{\text {disk }}$ are likely to come from differences in $c_{s}$ (i.e., warmer gas temperatures) and $\alpha_{\mathrm{SS}}$. This also indicates the approximative nature of our calculation.
The range of accretion rates between the stellar disks also widens over time, such that after $5000 \mathrm{yr}$ the total disk mass of the fastest-accreting halo from Stacy \& Bromm (2013) is a factor of ten greater than the total disk mass within our $z=$ 15 minihalo. To illustrate this, in Figure 11 we show the resulting evolution of the disk mass, as well as the mass of disks taken from the simulations of Stacy \& Bromm (2013) and Greif et al. (2012). In this figure, time is measured with respect to when the first sink forms. Note that the disk evolution of the green lines does not extend beyond a time of zero because these were taken from simulations which did not form sinks. The particular point that marks the transition from the stellar disk to the outer envelope is somewhat ambiguous, so to determine whether a gas particle is part of the disk we choose the simple criterion that it must have $n>10^{9} \mathrm{~cm}^{-3}$ and $f_{\mathrm{H}_{2}}>10^{-3}$. Therefore, only dense and molecular gas is included. Compared to other studies, the $z=15$ stellar disk grows at a very low rate. The slow overall growth rate of the mass of minihalo gas has translated to a slowly growing disk as well. The second-most slowly growing disk (green-dotted line in Figure 11) similarly belongs to the second-most slowly growing comparison minihalo.

We briefly note that as mass falls into the sink, a portion of the gas gets heated to the virial temperature $T_{\text {vir }}$ of the sink through release of gravitational potential (Figure 1). Given a sink mass of $0.2 M_{\odot}$ and using the accretion radius of $1.0 \mathrm{AU}$, we find a temperature of

$$
T_{\mathrm{vir}} \simeq \frac{G M_{\mathrm{sink}} m_{\mathrm{H}}}{k_{\mathrm{B}} r_{\mathrm{acc}}} \simeq 10^{4} \mathrm{~K} .
$$

This "warm bubble" of neutral gas expands at its sound speed of $c_{\mathrm{s}} \lesssim 10 \mathrm{~km} \mathrm{~s}^{-1}$. Thus, by $\sim 5000 \mathrm{yr}$ the warm bubble has reached a distances of approximately $c_{\mathrm{s}} t=10,000 \mathrm{AU}$, which corresponds to gas of density $n \sim 10^{7} \mathrm{~cm}^{-3}$. This warm phase of gas is visible in Figure 1 (see Turk et al. 2010 for further discussion of this warm and neutral gas phase).

These disk properties lead to very low sink accretion rates (Figure 12). The disk gas fragments to form a second and third sink 600 and $900 \mathrm{yr}$ after the first sink has formed, and two more between 3000 and 4000 yr. During the simulation two 

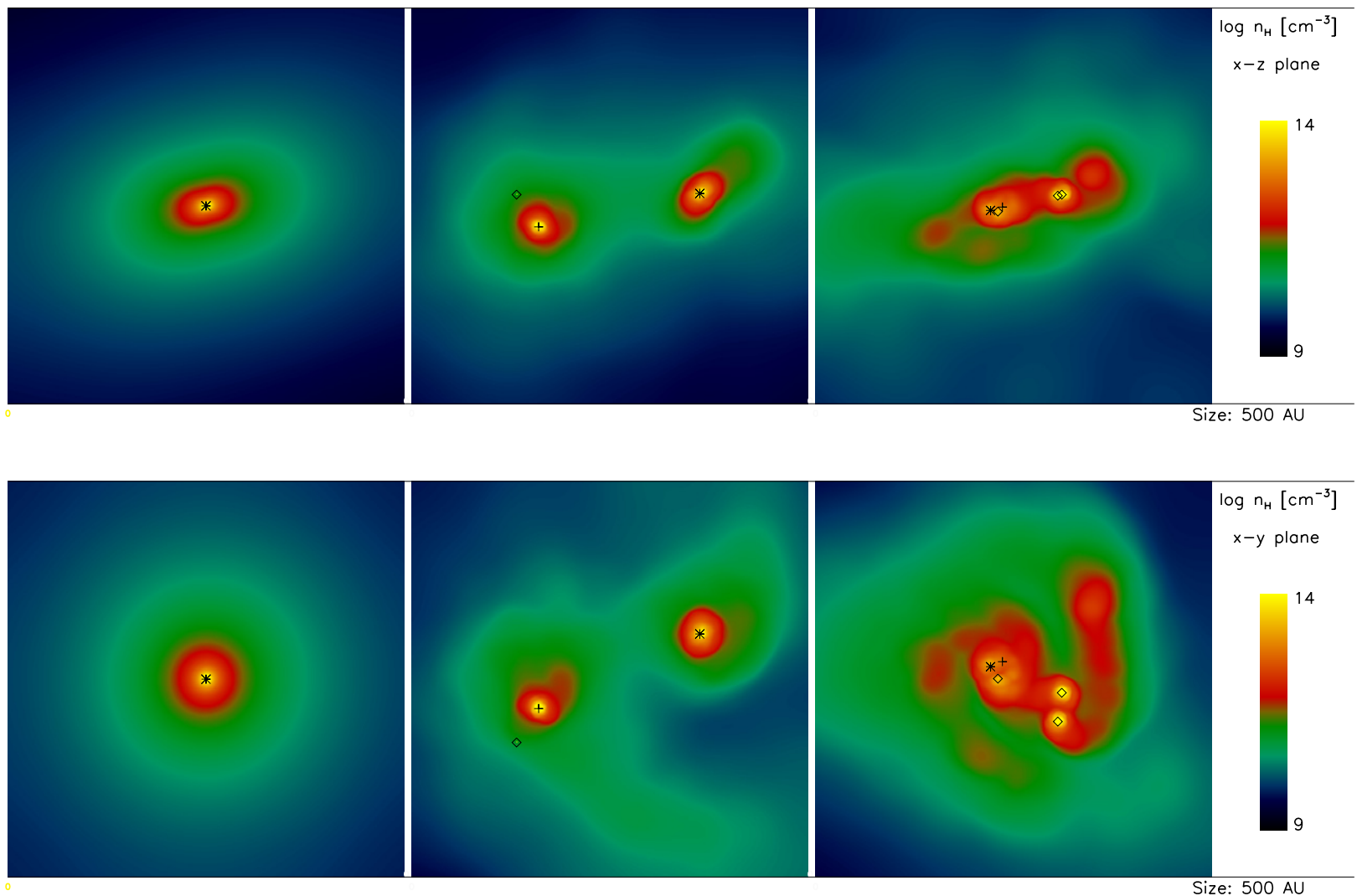

Figure 9. Density projection of central $500 \mathrm{AU}$ of gas around first sink. Top and bottom rows are the $x-z$ and $x-y$ planes, respectively. From left to right, times after sink formation are 3, 2000, and 3500 yr. The asterisk marks the largest sink. The plus symbol marks the second largest sink. The diamonds are other secondary sinks. Note the rapid changes in the sink orbital motion and the structure of the protostellar disk.

(A color version of this figure is available in the online journal.)

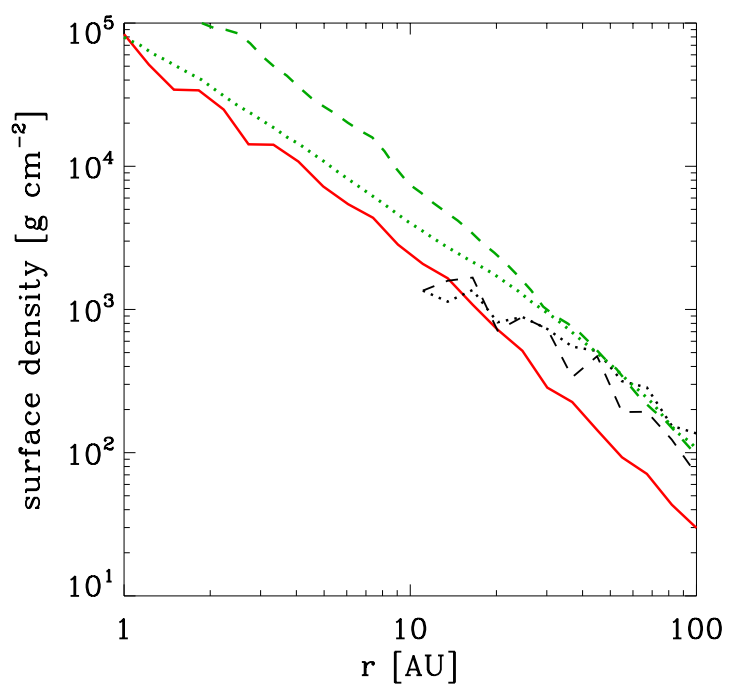

Figure 10. Surface density profile of this simulation (solid red line) as well as two selected minihalos from Stacy \& Bromm (2013; black dotted and dashed lines) and two from Greif et al. (2012; green lines). Profiles are shown just prior to the initial sink formation. Note that the Stacy \& Bromm (2013) calculations were resolved down to $\sim 10 \mathrm{AU}$, so we cannot calculate their surface densities on scales smaller than this.

(A color version of this figure is available in the online journal.)

other sinks form but quickly merge with the initial sink. A leastsquares power-low fit to the growth rate of the three largest sinks remaining at $t_{\mathrm{acc}} \sim 5000 \mathrm{yr}$ yields

$$
M_{*, 1} \simeq 0.61 M_{\odot}(t / 1000 \mathrm{yr})^{0.23}
$$

$$
\begin{aligned}
& M_{*, 2} \simeq 0.51 M_{\odot}(t / 1000 \mathrm{yr})^{0.28} \\
& M_{*, 3} \simeq 0.62 M_{\odot}(t / 1000 \mathrm{yr})^{0.32}
\end{aligned}
$$

Extending these power laws to $1 \mathrm{Myr}$, a typical accretion time for low-mass stars, these sinks would then reach 3.1, 3.5, and $5.7 M_{\odot}$. A similar approximation for the growth of the leastmassive sink predicts a late-time mass of $0.4 M_{\odot}$.

It is uncertain how many more protostars will form at later times and what masses they would reach, since we do not follow the simulation for sufficient time to track the longerterm evolution of the disk and surrounding $\sim 1000 M_{\odot}$ core as the protostars grow. As can be seen in Figure 7, the ratio of $M_{\text {enc }}$ to $M_{\mathrm{BE}}$ has a peak at $M_{\text {enc }} \lesssim 1000 M_{\odot}$ and then a rapid drop-off on larger scales. A similar drop off is seen at greater $M_{\text {enc }}$ for the more rapidly accreting halos. This central several hundred solar masses of material gravitationally infalls toward the central regions at a relatively slow rate of $\lesssim 10^{-3} M_{\odot} \mathrm{yr}^{-1}$, leading to little change in the outer $M_{\text {enc }}$ profile after $5000 \mathrm{yr}$ (see red dashed lines in Figure 7).

At the same time, the protostars followed in our simulation are not projected to become massive enough to develop an $\mathrm{H}$ II region that will blow away the gas. However, the luminosity and LW emission of the protostars will still serve to heat and stabilize the central gas, and the warm phase of the dense gas will continue to grow (Figure 1). We define the dense warm phase as gas which has $n>10^{9} \mathrm{~cm}^{-3}$ and $f_{\mathrm{H}_{2}}<10^{-3}$, such that only non-molecular gas is included. This phase approximately consists of $\sim 2 M_{\odot}$ of gas by the end of the simulation. The diversion of gas to the warm phase instead of the cool disk also helps to explain why $M_{\text {disk }}$ does not continue to increase 


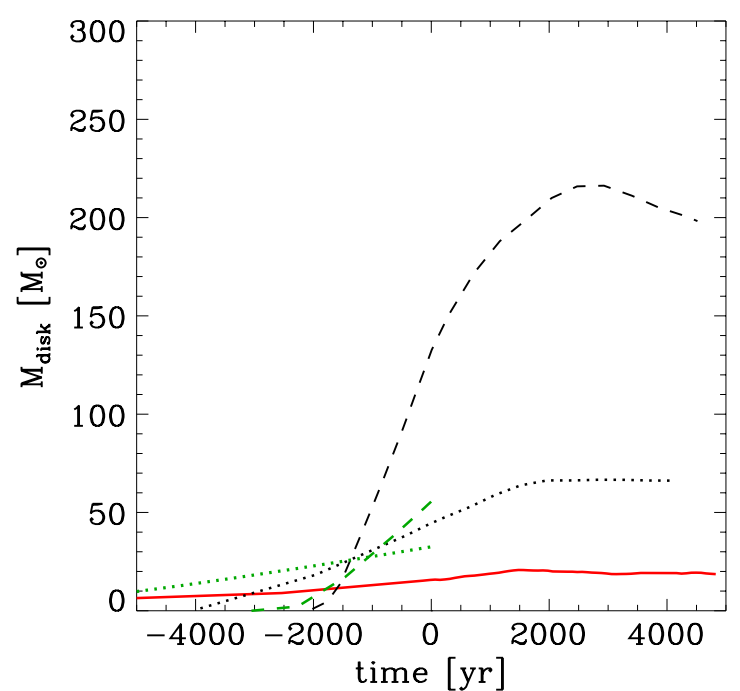

Figure 11. Evolution of disk mass over time. Time is measured with respect to the point at which a sink or protostar first forms. The solid red line represents the disk mass for our $z=15$ minihalo. The black dotted line represents the minihalo from Stacy \& Bromm (2013) that had the lowest overall stellar accretion rate, while the black dashed line represents that which had the greatest accretion rate. Green dotted and dashed lines additionally show the disk mass within two minihalos from Greif et al. (2012) which had the lowest and highest accretion rates, respectively.

(A color version of this figure is available in the online journal.)

above $\sim 20 M_{\odot}$ after $\sim 2000 \mathrm{yr}$ of sink accretion. Even with such feedback effects, however, we cannot rule out the possibility that over the subsequent $10^{5}$ to $10^{6} \mathrm{yr}$, gas inflow onto the disk will continue until a star reaches $\sim 10 M_{\odot}$ and ionizes its surroundings. We conjecture that stellar masses greater than $10 M_{\odot}$ will not be necessary to halt slow inflow like that seen in our simulation, and that even if we followed our simulation for very long times we still would not see a Pop III star reach the more typical masses of $50-100 M_{\odot}$. This will be confirmed with future numerical work.

\subsection{Evolution of Stellar Orbits}

During the disk evolution, the distance of the secondary sinks from the main sink ranges from $\sim 1$ AU to a few hundred AU.
In comparison, the occurrence of Roche-lobe overflow requires the size of one of the binary members to exceed its Roche-lobe radius $r_{L}$ :

$$
\frac{r_{L}}{a}=\max \left[0.46224\left(\frac{q}{1+q}\right)^{1 / 3}, 0.38+0.2 \log _{10} q\right]
$$

for $0<q<0.8$ (Paczyński 1971), where $q$ is the binary mass ratio, and $a$ is the semi-major axis. For $q=0.1$ we have $r_{L}=0.2 a$, while $q=1$ yields $r_{L}=0.38 a$. This brackets the expected range of mass ratios for our simulated binary system. For $a$ as small as $1 \mathrm{AU}$, we have $r_{L} \sim 3-6 \times 10^{12} \mathrm{~cm}$, or $40-80 R_{\odot}$. As AGB stars can reach well over $100 R_{\odot}$, this highlights the possibility that an AGB Pop III star may transfer mass to its companion.

This is a particularly interesting possibility for the lowestmass star, since it may experience additional close encounters as it orbits through and around the stellar system. If such a star remains below the "survival threshold" of $0.8 M_{\odot}$ (Figure 12), it may be observable in the present-day as a primordial AGBenriched star in the Milky Way halo or nearby dwarf galaxy. This smallest sink experiences close encounters with the largest sink at, e.g., 1500 and $2500 \mathrm{yr}$ (red line in the right panel of Figure 12) that nearly eject it from the disk. These encounters occur when the sink has grown to only $0.25 M_{\odot}$ and has a velocity of $\sim 5 \mathrm{~km} \mathrm{~s}^{-1}$ relative to the disk. This is not quite sufficient to escape the stellar system, however. It is uncertain how much more it will grow as it continues its orbit through the accretion disk, but it is still only $0.25 M_{\odot}$ at the end of the simulation and may remain below $1 M_{\odot}$ over its main-sequence lifetime (see also e.g., Johnson \& Khochfar 2011 for further discussion).

We emphasize the speculative nature of the above scenario. An AGB phase for the larger protostars of our system would not occur until $\sim 10^{8} \mathrm{yr}$. It is at this later time that the smallest protostar's orbit would need to come within $\gtrsim 1$ AU for mass transfer to occur. While its orbit over the first $5000 \mathrm{yr}$ ranges between one and a few hundred $\mathrm{AU}$, it remains uncertain how the orbit will evolve over the much longer AGB timescales, whether the star will undergo an ejection before this time, etc. However, in our simulation we do still see the basic initial requirements for our scenario: that a slowly accreting low-mass star is in close
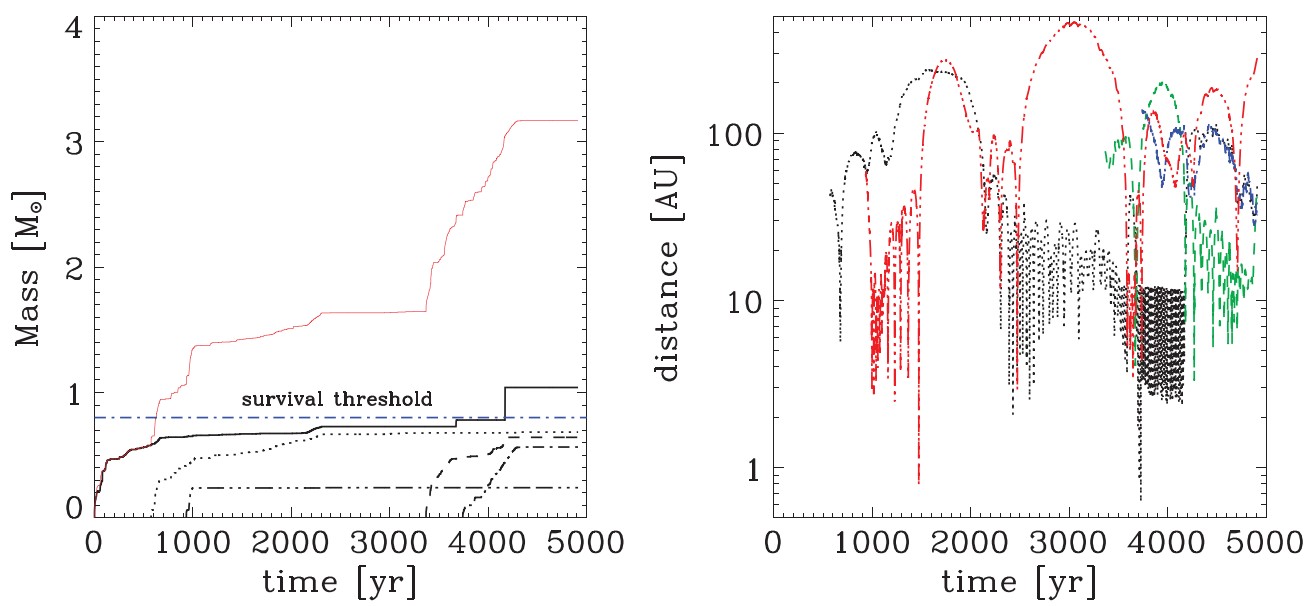

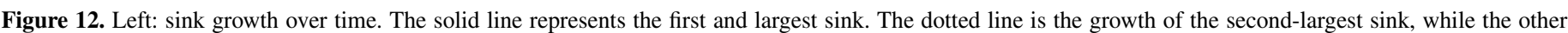

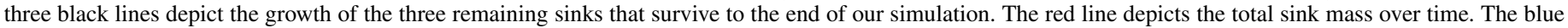

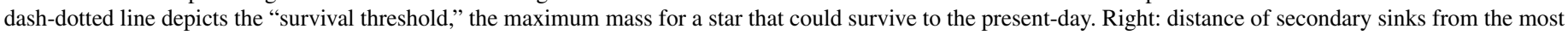
massive sink over time. Line styles refer to the same sinks as in the left panel, but with different colors for more visible contrast between lines.

(A color version of this figure is available in the online journal.) 

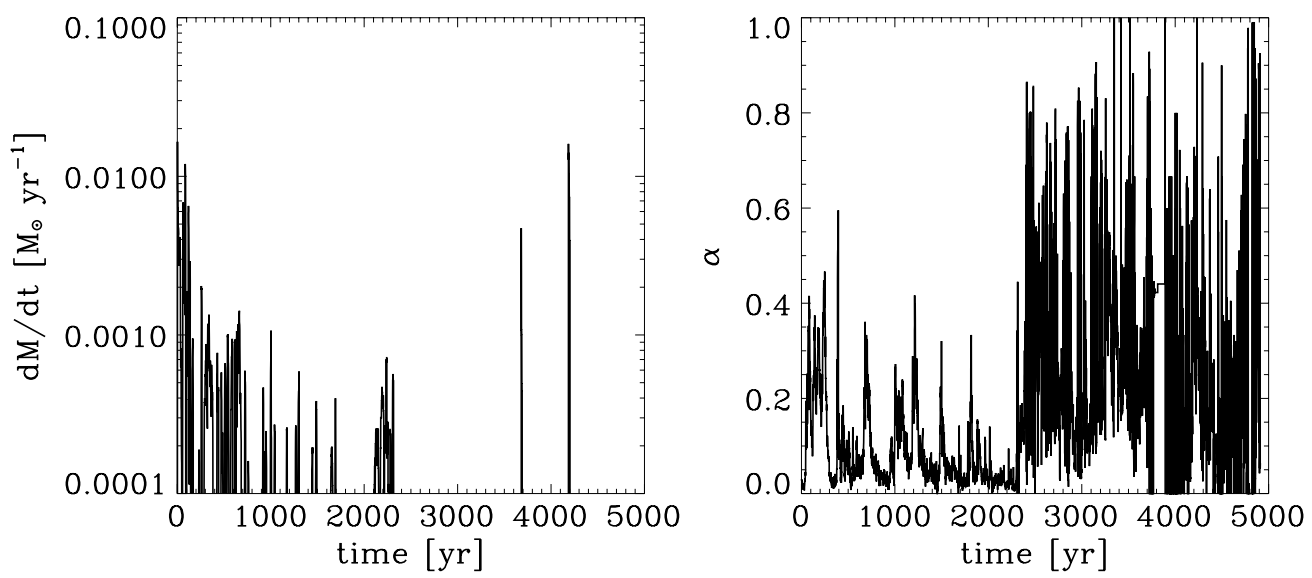

Figure 13. Evolution of various properties of near-sink gas: Left: accretion rate onto the main sink. Right: value of $\alpha$, where $\alpha=1$ corresponds to gas flowing radially toward the sink, while $\alpha=0$ corresponds to a rotationally dominated flow. The sink initially forms from an already disk-like gas configuration, so $\alpha$ is initially very low. Prior to $2500 \mathrm{yr}$, phases of more spherical motion around the sink generally correspond to periods of more rapid accretion.

orbit around a larger star on track to eventually undergo an AGB phase.

The variable orbital motion of the sinks further contributes to the high variability of the sink accretion rates. The accretion rate onto the main sink, shown in Figure 13, is nearly $\dot{M} \sim$ $10^{-2} M_{\odot} \mathrm{yr}^{-1}$ for the first few hundred years, but quickly drops to $\lesssim 10^{-3} M_{\odot} \mathrm{yr}^{-1}$ with periods where $\dot{M} \sim 0$. As the sink orbits through the stellar disk, the value of $\alpha$ is similarly variable, where $\alpha=1$ corresponds to radially dominated gas motion toward the sink and $\alpha=0$ corresponds to a rotationally dominated flow. Prior to $2500 \mathrm{yr}$, periods where $\alpha$ is closer to one corresponds to periods of more rapid accretion. In the latter half of the simulation when further disk fragmentation occurs, $\alpha$ becomes significantly more variable. On average, the main sink accretes at $\sim 2 \times 10^{-4} M_{\odot} \mathrm{yr}^{-1}$.

\section{INFLUENCE OF THE LYMAN-WERNER BACKGROUND}

\subsection{Overview}

A photodisssociating LW background built up by earlierforming Pop III stars may slow or prevent the cooling and collapse of the gas in our $z=15$ minihalo. However, the effect of such a background remains very uncertain. We first note that our analysis shows it is not simply the redshift but also the rotational structure of the gas that drives the unusually slow infall rate onto this minihalo. There is substantial variation in minihalo characteristics seen at all redshifts. This implies that such a highly rotationally supported, slowly accreting gas cloud may also exist within some $z=20-30$ minihalos.

When considering the effect of LW radiation, it is indeed appropriate to focus on the global background radiation, as opposed to radiation from a particular source. Given our box size, the nearest minihalo "outside" of the box would be $200 \mathrm{kpc}$ (comoving) away, or $\sim 20 \mathrm{kpc}$ (physical) away. Works by, e.g., Dijkstra et al. (2008) and Johnson et al. (2013) find that it is only rare high-density regions where LW flux from local sources will dominate over the global background, not regions like that in our simulation. However, the quickness with which this background will grow is very uncertain, and depends upon the early Pop III IMF. For instance, the semi-analytic models of Crosby et al. (2013) find that $J_{21}$ is at least $\sim 1$ at $z=15$, where $J_{21}$ represents units of $10^{-21} \mathrm{erg} \mathrm{s}^{-1} \mathrm{~cm}^{-2} \mathrm{~Hz}^{-1} \mathrm{sr}^{-1}$. The simulations of Johnson et al. (2013), on the other hand, find at

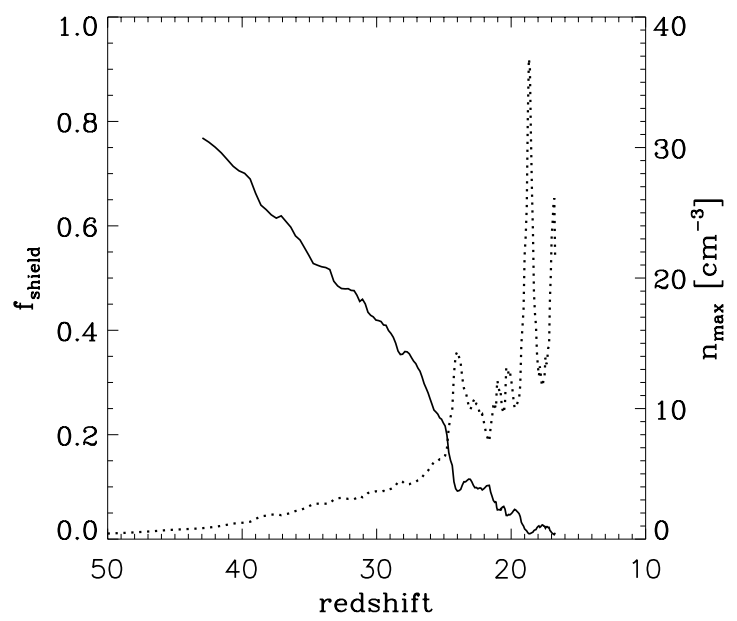

Figure 14. Evolution of $f_{\text {shield }}$ with redshift within the gas core of the minihalo. Dotted line also shows the maximum gas density $n_{\max }$.

this redshift that the overall $J_{21}$ is an order of magnitude lower, $J_{21} \sim 0.1$.

It is furthermore possible that the concurrently growing X-ray background from Pop III remnant black holes could have an opposing effect to the LW background. For instance, Jeon et al. (2012) find that the X-ray radiation from a high-mass BH binary can provide positive feedback such that gas collapse into distant minihalos is facilitated via $\mathrm{H}_{2}$ cooling promoted by the strong X-ray emission. In contrast, semianalytic models by, e.g., Tanaka et al. (2012) find that the X-ray heating of the IGM is a stronger effect than the associated enhancement of $\mathrm{H}_{2}$ formation in potential star-forming regions.

Simulations such as those in Machacek et al. (2003) and O'Shea \& Norman (2008), which found that the LW background delays gas collapse, did not account for the effect of $\mathrm{H}_{2}$ selfshielding. They furthermore assumed a $J_{21}$ that was constant instead of gradually evolving. To properly include the effects of this background, we would need to understand the still uncertain rate at which these backgrounds build up, and we also would need to account for $\mathrm{H}_{2}$ self-shielding within the minihalo.

The value of $f_{\text {shield }}$ represents the factor by which $\mathrm{H}_{2}$ absorption from the IGM and the outer parts of the minihalo will reduce the local LW flux within the inner star-forming parts of the minihalo. Figure 14 shows estimates of the average $f_{\text {shield }}$ 
within the gas core of the minihalo, defined as the central gas with densities within a factor of ten of the maximum gas density $n_{\text {max }}$. We estimate $f_{\text {shield }}$ in the same manner as in Johnson et al. (2013), in turn based upon Draine \& Bertoldi (1996) and Wolcott-Green et al. (2011):

$$
\begin{aligned}
f_{\text {shield }}\left(N_{\mathrm{H}_{2}}, T\right)= & \frac{0.965}{\left(1+x / b_{5}\right)^{1.1}}+\frac{0.035}{(1+x)^{0.5}} \\
& \times \exp \left[-8.5 \times 10^{-4}(1+x)^{0.5}\right],
\end{aligned}
$$

where $x \equiv N_{\mathrm{H}_{2}} / 5 \times 10^{14} \mathrm{~cm}^{-2}, b_{5} \equiv b / 10^{5} \mathrm{~cm} \mathrm{~s}^{-1}$, and $b$ is the Doppler broadening parameter given by $b \equiv\left(k_{\mathrm{B}} T / m_{\mathrm{H}}\right)^{1 / 2}$ (see Johnson et al. 2013 for further details). By $z \lesssim 25, f_{\text {shield will }}$ reduce the local LW flux by over an order of magnitude, greatly helping to reduce any possible effects of the LW background.

We finally note that we do not argue that Pop III stars were typically low mass, but that in rare environments, such a lowmass formation mode could occur. This is potentially important as it would allow observers to detect such Pop III fossils as surviving stars in our local cosmic neighborhood (stellar archaeology). The standard picture to date has been that Pop III stars always grow to masses that would have led to their death a long time ago. Because the strength of the LW background is subject to huge uncertainties, including spatial fluctuations and local opacity, conditions such as those simulated here cannot be excluded.

\subsection{Numerical Tests}

We further numerically examine LW effects with a set of re-simulations of the initial minihalo collapse, beginning from $z=50$, but this time including a LW background. We follow the gas collapse up to the point just before sink formation ( $n=10^{16} \mathrm{~cm}^{-3}$ ), but we did not have sufficient computational resources to follow the gas evolution further. The LW background grows with time as

$$
J_{21}=J_{21,0} \times 10^{-\left(z-z_{0}\right) / 5},
$$

where $J_{21,0}$ is a normalization parameter set to range from 0.1 to 10 , and $z_{0}=10$. When $J_{21,0}=1$, we obtain a good fit to the LW background evolution presented in Figure 1 of Greif \& Bromm (2006; see also Pawlik et al. 2013). From this we apply a photo-dissociation rate of

$$
k_{\mathrm{H}_{2}}=1.38 \times 10^{-12} f_{\text {shield }} J_{21} \mathrm{~s}^{-1} .
$$

We find that the LW background initially serves to delay minihalo collapse. For the more extreme $J_{21,0}=10$ case, the minihalo gas still has not collapsed at $z=13.3$. At this time the densest gas has densities of only $n=10 \mathrm{~cm}^{-3}$. To save computational time we did not follow this simulation further. However, we conclude that at such high $J_{21,0}$ values the gas evolution is significantly altered. Gas collapse will likely occur only once the minihalo reaches higher mass and virial temperatures of $\sim 10^{4} \mathrm{~K}$. Gas fragmentation may be suppressed, and the gas may collapse directly into a black hole (see, e.g., Oh \& Haiman 2002; Bromm \& Loeb 2003).

The gas evolution is much less affected for the more physically realistic value of $J_{21,0}=0.1$, in which case collapse to sink particle densities of $n=10^{16} \mathrm{~cm}^{-3}$ is delayed by $1.7 \times 10^{6} \mathrm{yr}$. In Figure 15 we show the state of the gas at the point that the gas has reached $n=10^{16} \mathrm{~cm}^{-3}$ in each test simulation. For $J_{21,0}=0.1$ (solid black line in Figure 15), the gas profile does not significantly differ from the fiducial $J_{21,0}=0$ case (red line in Figure 15). As expected, outside of $10^{5} \mathrm{AU}$ the $\mathrm{H}_{2}$ fraction is reduced due to the LW background, while shielding is effective in the more central regions and even allows for a slightly larger $\mathrm{H}_{2}$ fraction inside of $10^{5} \mathrm{AU}$. In the inner $1000 \mathrm{AU}$, the gas properties do not differ by more than a few tens of percent, though the central densities and estimated spherical accretion rate (see Equation (14)) are somewhat enhanced. The enclosed mass at all given radii, as well as the ratio of $M_{\text {enc }}$ to $M_{\mathrm{BE}}$, are also slightly larger (Figure 16). This leads to a slightly higher overall disk mass as the gas approaches $n=10^{16} \mathrm{~cm}^{-3}$, when $M_{\text {disk }} \sim 20 M_{\odot}$ as opposed to $\sim 16 M_{\odot}$ in the fiducial case (Figure 17). Note, however, that these values of $M_{\text {disk }}$ and $\dot{M}_{\text {sphere }}$ are approximately half to a tenth as large as those found in the other minihalos discussed in Section 4.2. Thus, under a small $J_{21,0}=0.1$ background we would still likely find an unusually low-mass Pop III system, though probably more massive than the fiducial case.

For $J_{21,0}=1$, the differences are more significant (dashed line in Figure 15). Collapse is delayed by $3.3 \times 10^{7} \mathrm{yr}$ before the gas finally reaches the sink density of $n=10^{16} \mathrm{~cm}^{-3}$. The $\mathrm{H}_{2}$ fraction is approximately half of that found in the fiducial case at distances greater than $10^{4} \mathrm{AU}$. However, inside $10^{4} \mathrm{AU}$ shielding becomes very effective, and the $\mathrm{H}_{2}$ increases more rapidly than the other test cases as the radius declines. In the central $10^{4} \mathrm{AU}$ the gas density, sound speed, and infall velocity are all reduced, and $\dot{M}_{\text {sphere }}$ is up to an order of magnitude below the fiducial case. The enclosed mass at all given radii is also reduced by up to a factor of a few (Figure 16). The central $30 M_{\mathrm{enc}}$ has a higher ratio of $M_{\mathrm{enc}}$ to $M_{\mathrm{BE}}$, but outside of this region the ratio is much reduced and the gas is more gravitationally stable. Overall, this leads to $M_{\text {disk }} \sim 5 M_{\odot}$ when $n=10^{16} \mathrm{~cm}^{-3}$, several times smaller than the other cases.

When comparing these simulations at the same peak densities, but not at the same physical time, the effect of increasing the $\mathrm{LW}$ background is not monotonic. For the more realistic values of $J_{21,0}=0.1$ or 1 , we find central accretion rates that are either slightly enhanced or significantly reduced. In these cases the LW background does not seem to change our general finding of a minihalo which hosts a system of very slowly accreting Pop III stars.

\section{DISCUSSION AND CONCLUSIONS}

We present a three-dimensional simulation of the formation and growth of a Pop III stellar system. This calculation was initialized on cosmological scales while resolving lengths as small as 1 AU. We found that the host minihalo formed at an unusually low redshift of $z=15$, leading to a low DM and baryonic accretion rate as well as a low-mass and slowly growing stellar system. The stars in the system can be expected to reach $\sim 0.5$ to $5 M_{\odot}$ after $1 \mathrm{Myr}$. This is nearly an order of magnitude slower than rates typically found within $z \gtrsim 20$ stellar systems (e.g., Stacy et al. 2010; Greif et al. 2011; Smith et al. 2011). We additionally find that a LW background as high as $J_{21,0}=1$ will not significantly change our finding of uncharacteristically small infall rates onto the central star-forming region of our minihalo.

It is uncertain how common such low-mass Pop III systems will be. The minihalo we present here hosts the slowestaccreting Pop III system of approximately 10 minihalos which are included in our comparison in Section 4. Hirano et al. (2014) use approximately 100 minihalos from cosmological simulations to initialize two-dimensional simulations of Pop III 

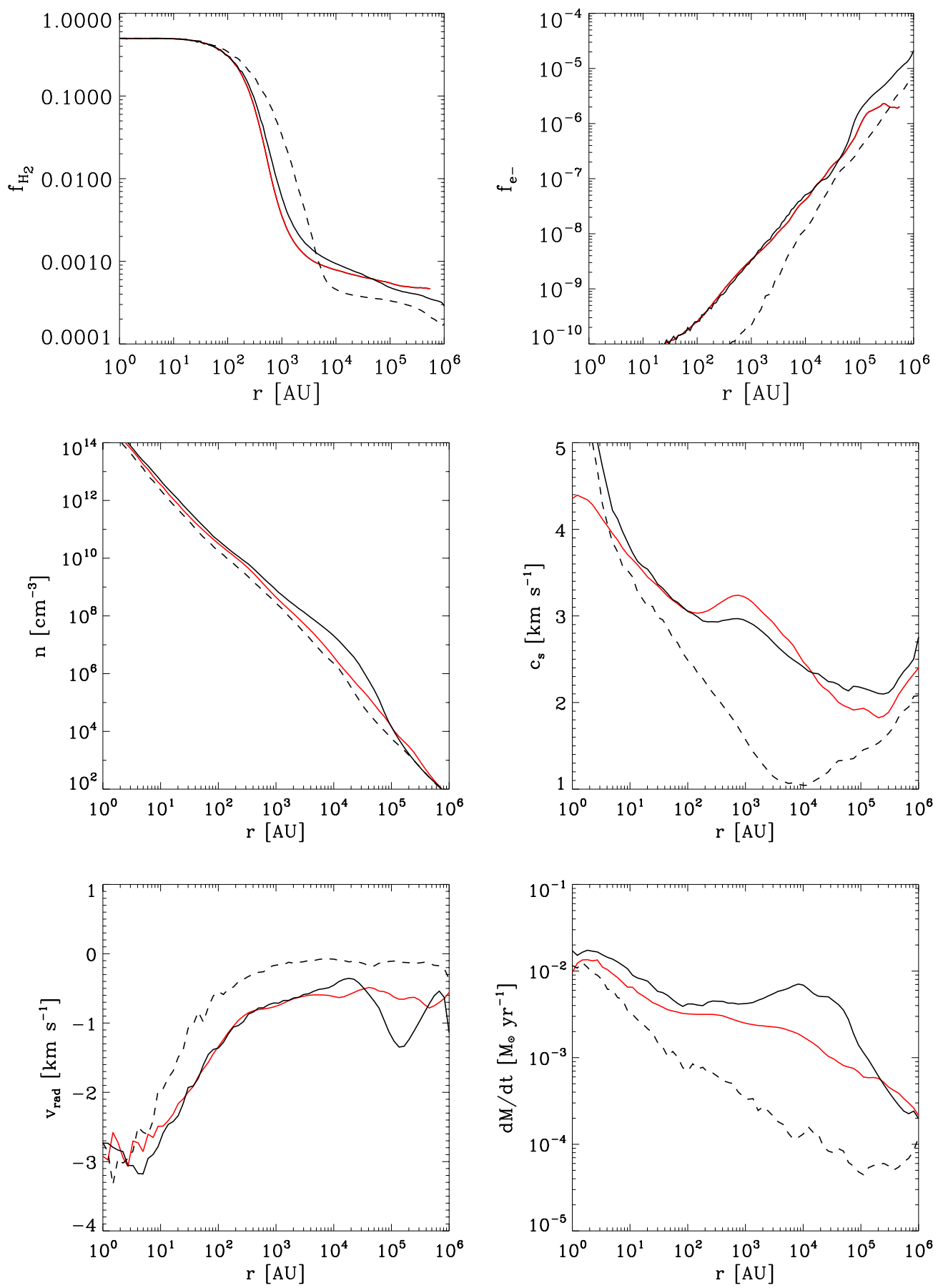

Figure 15. Radial profiles of the central gas within our simulated minihalo under various strengths of LW background, measured when the gas has reached a density of $n=10^{16} \mathrm{~cm}^{-3}$ in the respective simulations. The solid black lines denote the $J_{21,0}=0.1$ case, while dashed lines represent $J_{21,0}=1$. The red lines are taken from the fiducial $J_{21,0}=0$ simulation discussed throughout this work. Top left: $\mathrm{H}_{2}$ fraction. Top right: electron fraction. Middle left: number density. Middle right: sound speed. Bottom left: radial infall velocity. Bottom right: estimated spherical accretion rate ( $\dot{M}_{\text {sphere }}$; see Equation (14)). The gas profiles are not significantly altered for $J_{21,0}=0.1$, though density, infall velocity, and $\dot{M}_{\text {sphere }}$ are slightly enhanced. The $J_{21,0}=1$ background surprisingly leads to the generally opposite effect of reduced densities, sound speeds, and infall rates.

(A color version of this figure is available in the online journal.)

stellar growth under feedback. Only one star can be followed per minihalo, and in this case their smallest star is expected to grow to $\sim 9 M_{\odot}$. Were they able to follow fragmentation within this particular minihalo, it is possible that the stellar mass may have instead been distributed among several stars of lower mass. We thus make a very rough estimate that one out of a few tens to one out of 100 minihalos will host a low-mass system similar to what we find in our simulation. Considering that the mass of $10^{5}-10^{6}$ minihalos will ultimately become incorporated into a Milky Way-type galaxy, it is conceivable that on the order of thousands of Pop III stars from such low-mass systems may exist in the nearby Galactic halo.

Further study will be necessary to determine more precisely how common such low-mass Pop III systems are and whether our $z=15$ Pop III system indicates a more general transition in the Pop III IMF from $z \sim 30$ to $\sim 10$, or if our particular 

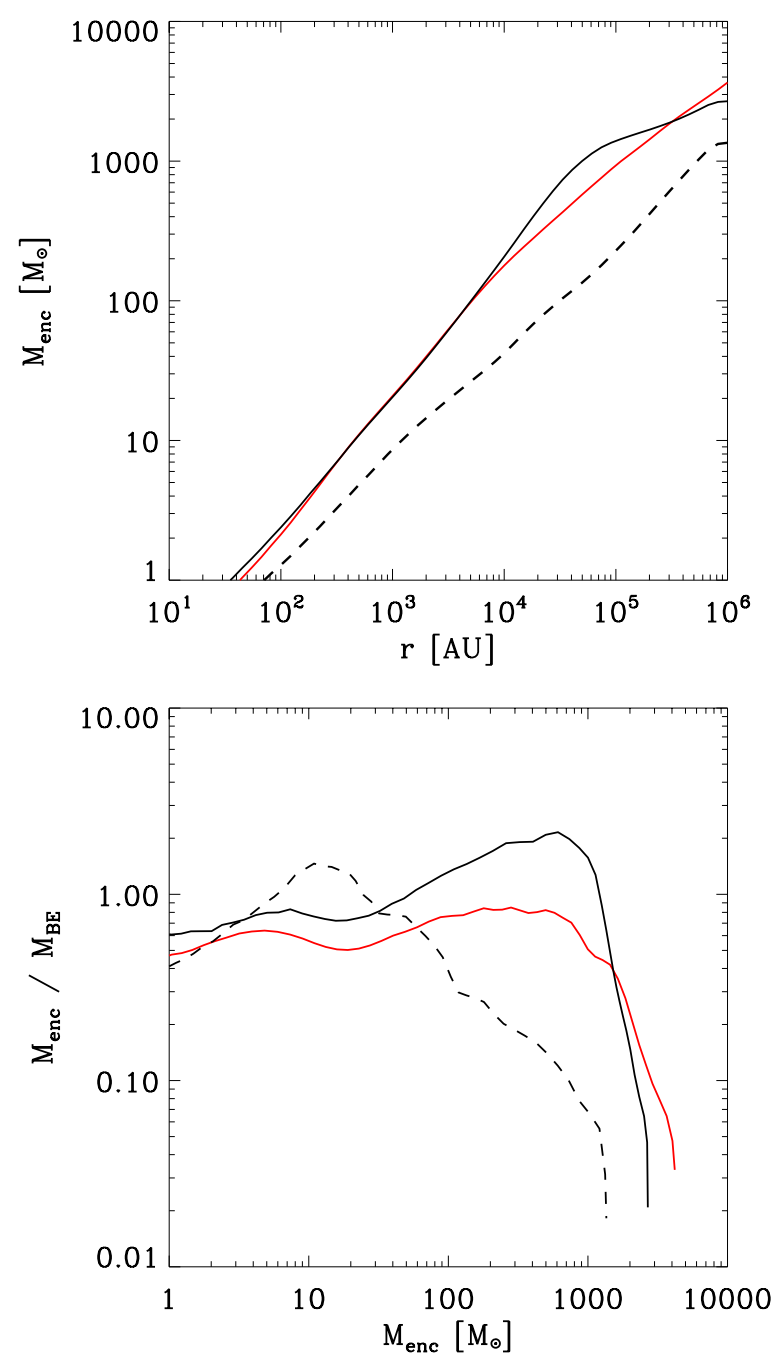

Figure 16. Top: enclosed mass $M_{\text {enc }}$ vs. radius for our test cases at the point when the maximum gas density first reaches $10^{16} \mathrm{~cm}^{-3}$. The solid black lines denote the $J_{21,0}=0.1$ case, while dashed lines represent $J_{21,0}=1$. The red lines are taken from the fiducial $J_{21,0}=0$ simulation discussed throughout this work. The central point is taken to be the most dense gas particle. Bottom: ratio of $M_{\mathrm{enc}}$ to $M_{\mathrm{BE}}$ vs. $M_{\mathrm{enc}}$. Lines have same meaning as in the upper panel. While the $J_{21,0}=0.1$ case has slightly enhanced mass and $M_{\text {enc }}$ to $M_{\mathrm{BE}}$ ratio, these are somewhat reduced for the $J_{21,0}=1$ case.

(A color version of this figure is available in the online journal.)

minihalo was an unusual case even for its low redshift. While minihalo comparisons of other works do not find this same transition in accretion rate (Gao et al. 2007; O'Shea \& Norman 2007), the numerical analyses by de Souza et al. (2013) do find a transition in the spin distribution of gas within minihalos as redshift declines. According to the semi-analytic model of McKee \& Tan (2008), such differences in rotational support will in turn lead to differences in the protostellar feedback and the final mass reached by the protostar. de Souza et al. (2013) argue that this will cause the Pop III IMF peak to shift to lower mass with lower redshift. This remains to be tested with more physically detailed simulations.

Understanding the Pop III IMF evolution with redshift will additionally require a greater knowledge of how the buildup of global background radiation as well as magnetic fields proceeded at this redshift (e.g., Schleicher et al. 2010; Schober et al. 2012; Turk et al. 2012). Understanding how the IMF evolved to later times is particularly important given that recent

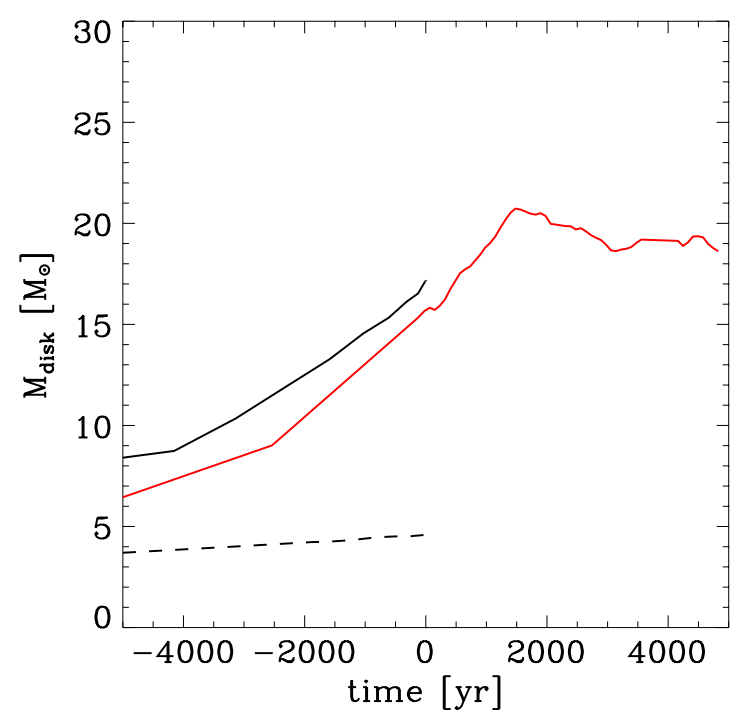

Figure 17. Evolution of disk mass over time for $J_{21,0}=0, J_{21,0}=0.1$, and $J_{21,0}=1$. Line styles have the same meaning as in the previous figure. Time is measured with respect to the time when the gas first reaches $n=10^{16} \mathrm{~cm}^{-3}$. The $J_{21,0}=0.1$ background leads to a slightly enhanced $M_{\text {disk }}$ as compared with the fiducial $J_{21,0}=0$ model. The larger $J_{21,0}=1$ background in fact leads to $M_{\text {disk }}$ which is consistently several times smaller than the fiducial case. Realistic LW backgrounds are therefore unlikely to change our overall finding of unusually low Pop III accretion rates within our simulated minihalo.

(A color version of this figure is available in the online journal.)

work has indicated that metal-free gas will indeed survive to relatively low redshift. For instance, Simcoe et al. (2012) reported observations of extremely low metallicity or possibly metal-free gas within a $z \sim 7$ damped Ly $\alpha$ system, while Fumagalli et al. (2011) reported the detection of metal-free gas within Lyman-limit systems at $z \gtrsim 3$. Numerical work (e.g., Muratov et al. 2013; see also Scannapieco et al. 2005) has similarly indicated that Pop III star formation can continue to $z \sim 6$. Our preliminary tests presented in Section 5, however, indicate that for a range of LW backgrounds our Pop III system will still undergo unusually low accretion rates.

AGB stars are important to understanding the early chemical evolution of the galaxy (e.g., Karakas 2010; Campbell \& Lattanzio 2008; Karakas \& Lugaro 2010). They are known to produce significant quantities of carbon, nitrogen, and s-process elements. (Busso et al. 2001; Siess et al. 2002; Siess \& Goriely 2003). They may also help to explain the observation of large amounts of dust in high- $z$ galaxies and quasars, which implies rapid dust production in the early universe (Bertoldi et al. 2003; Valiante et al. 2009; Gall et al. 2011). Our results reveal a pathway for such AGB star formation within primordial gas. In particular, the larger 3-5 $M_{\odot}$ stars of our simulation are sufficiently short-lived to undergo a metal and dust-enriching AGB phase by $z>6$, while the smaller $\lesssim 1 M_{\odot}$ star is long-lived enough that it may still be observed as carrying the enrichment signatures of its larger companions. The physical scenario suggested by our simulations may indeed explain the abundances of certain metal-poor stars in the Milky Way halo, particularly those with unusual features in $\mathrm{C}, \mathrm{N}$, and $\mathrm{O}$. Studies have found that some of these may be Pop III stars which received material from an AGB companion that is now a white dwarf (e.g., Suda et al. 2004, 2013).

Studies over recent years have found increasing complexity in the nature of Pop III stars. Though dust and metallicity by definition do not play a role in primordial star formation, 
other physical processes such as multiplicity (e.g., Clark et al. 2008; Stacy et al. 2010; Greif et al. 2011) as well as the binary nature and rotation rate of Pop III stars (e.g., Stacy \& Bromm 2013) will also be of crucial importance. In addition, feedback (e.g., Hosokawa et al. 2011; Smith et al. 2011; Stacy et al. 2012) and magnetic fields play a central role in Pop III protostellar accretion. However, our work shows that even when such processes are not included in simulations, the differences in minihalo environments alone can lead to substantial variation between primordial stellar clusters. As shown by Jappsen et al. (2009; see also Dopcke et al. 2013), our work demonstrates that the transition to a low-mass IMF will depend upon not only a critical metallicity (e.g., Bromm et al. 2001), but also upon the characteristics of the primordial star-forming region.

We thus predict the rare existence of low-mass Pop III stars that have survived until the present day and that may show evidence of enrichment from a companion's AGB-phase mass overflow. Continued improvements in simulation techniques and computational power, as well as constraints provided by observations such as abundance measurements of metal-poor stars in the Galactic halo and dwarf galaxies (e.g., Beers \& Christlieb 2005; Frebel et al. 2005; Caffau et al. 2011; see also Karlsson et al. 2013), will provide an increasingly refined picture of the role Pop III stars played in shaping the early universe. Ultimately, the question of whether true Pop III survivors exist, e.g., in the guise of AGB self-enrichment as suggested in this paper, is a question that can be tested empirically. The potential for such stellar archaeological constraints is demonstrated by the exciting recent discovery by the SkyMapper Southern Sky Survey of a star with no detected Fe-peak elements, but low abundances of C, N, and O (Keller et al. 2014). Indeed, the detected abundance pattern closely resembles an AGB self-enrichment pattern with the one crucial exception of an extremely low, but non-zero, $\mathrm{Ca}$ abundance. The latter cannot be accommodated with AGB enrichment, but instead points to the signature of supernova enrichment from a massive Pop III progenitor, rendering this extreme star second-generation. However, the search for Pop III fossils is clearly within the reach of current and upcoming surveys.

The authors thank the anonymous referee who helped them improve this manuscript. A.S. is grateful for support from the JWST Postdoctoral Fellowship through the NASA Postdoctoral Program (NPP). V.B. acknowledges support from NASA through Astrophysics Theory and Fundamental Physics Program grant NNX09AJ33G and from NSF through grant AST1009928. Resources supporting this work were provided by the NASA High-End Computing (HEC) Program through the NASA Advanced Supercomputing (NAS) Division at Ames Research Center.

\section{REFERENCES}

Abel, T., Anninos, P., Zhang, Y., \& Norman, M. L. 1997, NewA, 2, 181

Abel, T., Bryan, G. L., \& Norman, M. L. 2002, Sci, 295, 93

Alvarez, M. A., Bromm, V., \& Shapiro, P. R. 2006, ApJ, 639, 621

Bate, M. R., Bonnell, I. A., \& Price, N. M. 1995, MNRAS, 277, 362

Bate, M. R., \& Burkert, A. 1997, MNRAS, 288, 1060

Beers, T. C., \& Christlieb, N. 2005, ARA\&A, 43, 531

Bertoldi, F., Carilli, C. L., Cox, P., et al. 2003, A\&A, 406, L55

Bromm, V. 2013, RPPh, 76, 112901

Bromm, V., Coppi, P. S., \& Larson, R. B. 2002, ApJ, 564, 23

Bromm, V., Ferrara, A., Coppi, P. S., \& Larson, R. B. 2001, MNRAS, 328, 969

Bromm, V., \& Loeb, A. 2003, ApJ, 596, 34

Bromm, V., \& Loeb, A. 2004, NewA, 9, 353

Bromm, V., Yoshida, N., \& Hernquist, L. 2003, ApJL, 596, L135
Busso, M., Gallino, R., Lambert, D. L., Travaglio, C., \& Smith, V. V. 2001, ApJ 557,802

Caffau, E., et al. 2011, Natur, 477, 67

Campbell, S. W., \& Lattanzio, J. C. 2008, A\&A, 490, 769

Chatzopoulos, E., \& Wheeler, J. C. 2012, ApJ, 748, 42

Cherchneff, I., \& Dwek, E. 2010, ApJ, 713, 1

Clark, P. C., Glover, S. C. O., \& Klessen, R. S. 2008, ApJ, 672, 757

Clark, P. C., Glover, S. C. O., Klessen, R. S., \& Bromm, V. 2011a, ApJ, 727,110

Clark, P. C., Glover, S. C. O., Smith, R. J., et al. 2011b, Sci, 331, 1040

Crosby, B. D., O’Shea, B. W., Smith, B. D., Turk, M. J., \& Hahn, O. 2013, ApJ, 773,108

Dekel, A., Birnboim, Y., Engel, G., et al. 2009, Natur, 457, 451

de Souza, R. S., Ciardi, B., Maio, U., \& Ferrara, A. 2013, MNRAS, 428, 2109

Dijkstra, M., Haiman, Z., Mesinger, A., \& Wyithe, J. S. B. 2008, MNRAS, 391, 1961

Dopcke, G., Glover, S. C. O., Clark, P. C., \& Klessen, R. S. 2013, ApJ, 766, 103

Draine, B. T., \& Bertoldi, F. 1996, ApJ, 468, 269

Faucher-Giguère, C.-A., Kereš, D., \& Ma, C.-P. 2011, MNRAS, 417, 2982

Frebel, A., Aoki, W., Christlieb, N., et al. 2005, Natur, 434, 871

Frommhold, L. 1994, Collision-induced Absorption in Gases (Cambridge: Cambridge Univ. Press)

Fumagalli, M., O’Meara, J. M., \& Prochaska, J. X. 2011, Sci, 334, 1245

Gall, C., Hjorth, J., \& Andersen, A. C. 2011, A\&ARv, 19, 43

Gao, L., White, S. D. M., Jenkins, A., Frenk, C. S., \& Springel, V. 2005, MNRAS, 363,379

Gao, L., Yoshida, N., Abel, T., et al. 2007, MNRAS, 378, 449

Genel, S., Genzel, R., Bouché, N., et al. 2008, ApJ, 688, 789

Greif, T., Springel, V., White, S., et al. 2011, ApJ, 737, 75

Greif, T. H., \& Bromm, V. 2006, MNRAS, 373, 128

Greif, T. H., Bromm, V., Clark, P. C., et al. 2012, MNRAS, 424, 399

Greif, T. H., Glover, S. C. O., Bromm, V., \& Klessen, R. S. 2010, ApJ, 716, 510

Greif, T. H., Johnson, J. L., Bromm, V., \& Klessen, R. S. 2007, ApJ, 670, 1

Greif, T. H., Johnson, J. L., Klessen, R. S., \& Bromm, V. 2009, MNRAS, 399,639

Greif, T. H., Springel, V., \& Bromm, V. 2013, MNRAS, 434, 3408

Haiman, Z., Thoul, A. A., \& Loeb, A. 1996, ApJ, 464, 523

Hansen, C. J., Kawaler, S. D., \& Trimble, V. 2004, Stellar Interiors: Physical Principles, Structure, and Evolution (New York: Springer)

Hartmann, L., Cassen, P., \& Kenyon, S. J. 1997, ApJ, 475, 770

Heger, A., \& Woosley, S. E. 2002, ApJ, 567, 532

Henyey, L. G., Lelevier, R., \& Levée, R. D. 1955, PASP, 67, 154

Hirano, S., Hosokawa, T., Yoshida, N., et al. 2014, ApJ, 781, 60

Hirano, S., \& Yoshida, N. 2013, ApJ, 763, 52

Hosokawa, T., Omukai, K., Yoshida, N., \& Yorke, H. W. 2011, Sci, 334, 1250

Hosokawa, T., Yorke, H. W., \& Omukai, K. 2010, ApJ, 721, 478

Jappsen, A.-K., Klessen, R. S., Glover, S. C. O., \& Mac Low, M.-M. 2009, ApJ, 696, 1065

Jeon, M., Pawlik, A. H., Greif, T. H., et al. 2012, ApJ, 754, 34

Johnson, J. L., Dalla, V. C., \& Khochfar, S. 2013, MNRAS, 428, 1857

Johnson, J. L., Greif, T. H., \& Bromm, V. 2007, ApJ, 665, 85

Johnson, J. L., \& Khochfar, S. 2011, MNRAS, 413, 1184

Karakas, A. I. 2010, MNRAS, 403, 1413

Karakas, A. I., \& Lugaro, M. 2010, PASA, 27, 227

Karlsson, T., Bromm, V., \& Bland-Hawthorn, J. 2013, RvMP, 85, 809

Kashlinsky, A., \& Rees, M. J. 1983, MNRAS, 205, 955

Keller, S. C., Bessell, M. S., Frebel, A., et al. 2014, Natur, 506, 463

Kitayama, T., Yoshida, N., Susa, H., \& Umemura, M. 2004, ApJ, 613, 631

Lacey, C., \& Cole, S. 1993, MNRAS, 262, 627

Latif, M. A., Schleicher, D. R. G., Schmidt, W., \& Niemeyer, J. 2013, ApJL, 772, L3

Loeb, A. 2010, How Did the First Stars and Galaxies Form? (Princeton, NJ: Princeton Univ. Press)

Machacek, M. E., Bryan, G. L., \& Abel, T. 2003, MNRAS, 338, 273

Madau, P., Ferrara, A., \& Rees, M. J. 2001, ApJ, 555, 92

Maio, U., Khochfar, S., Johnson, J. L., \& Ciardi, B. 2011, MNRAS, 414, 1145

Martel, H., Evans, N. J., \& Shapiro, P. R. 2006, ApJS, 163, 122

McKee, C. F., \& Tan, J. C. 2008, ApJ, 681, 771

Mo, H. J., \& White, S. D. M. 1996, MNRAS, 282, 347

Mori, M., Ferrara, A., \& Madau, P. 2002, ApJ, 571, 40

Muratov, A. L., Gnedin, O. Y., Gnedin, N. Y., \& Zemp, M. 2013, ApJ, 773, 19

Nakamura, F., \& Umemura, M. 2001, ApJ, 548, 19

Navarro, J. F., \& White, S. D. M. 1994, MNRAS, 267, 401

Neistein, E., van den Bosch, F. C., \& Dekel, A. 2006, MNRAS, 372, 933

Nomoto, K., Maeda, K., Umeda, H., et al. 2003, PThPS, 151, 44

Norman, M. L., O'Shea, B. W., \& Paschos, P. 2004, ApJL, 601, L115

Oh, S. P., \& Haiman, Z. 2002, ApJ, 569, 558 
Omukai, K., \& Palla, F. 2003, ApJ, 589, 677

O'Shea, B. W., \& Norman, M. L. 2007, ApJ, 654, 66

O'Shea, B. W., \& Norman, M. L. 2008, ApJ, 673, 14

Paczyński, B. 1971, ARA\&A, 9, 183

Pawlik, A. H., Milosavljević, M., \& Bromm, V. 2013, ApJ, 767, 59

Press, W. H., \& Schechter, P. 1974, ApJ, 187, 425

Prialnik, D., \& Livio, M. 1985, MNRAS, 216, 37

Reed, D. S., Bower, R., Frenk, C. S., et al. 2005, MNRAS, 363, 393

Ripamonti, E., \& Abel, T. 2004, MNRAS, 348, 1019

Ripamonti, E., Haardt, F., Ferrara, A., \& Colpi, M. 2002, MNRAS, 334,401

Scannapieco, C., Tissera, P. B., White, S. D. M., \& Springel, V. 2005, MNRAS, 364,552

Schleicher, D. R. G., Banerjee, R., Sur, S., et al. 2010, A\&A, 522, A115

Schober, J., Schleicher, D., Federrath, C., et al. 2012, ApJ, 754, 99

Shakura, N. I., \& Sunyaev, R. A. 1973, A\&A, 24, 337

Shu, F. H. 1977, ApJ, 214, 488

Siess, L., \& Goriely, S. 2003, NuPhA, 718, 524

Siess, L., Livio, M., \& Lattanzio, J. 2002, ApJ, 570, 329

Simcoe, R. A., Sullivan, P. W., Cooksey, K. L., et al. 2012, Natur, 492, 79

Smith, R. J., Glover, S. C. O., Clark, P. C., Greif, T., \& Klessen, R. S. 2011, MNRAS, 414, 3633

Smith, R. J., Hosokawa, T., Omukai, K., Glover, S. C. O., \& Klessen, R. S. 2012, MNRAS, 424, 457

Sokasian, A., Yoshida, N., Abel, T., Hernquist, L., \& Springel, V. 2004, MNRAS, 350,47

Springel, V. 2005, MNRAS, 364, 1105
Stacy, A., \& Bromm, V. 2013, MNRAS, 433, 1094

Stacy, A., Greif, T. H., \& Bromm, V. 2010, MNRAS, 403, 45

Stacy, A., Greif, T. H., \& Bromm, V. 2012, MNRAS, 422, 290

Stahler, S. W., Palla, F., \& Salpeter, E. E. 1986a, ApJ, 308, 697

Stahler, S. W., Palla, F., \& Salpeter, E. E. 1986b, ApJ, 302, 590

Suda, T., Aikawa, M., Machida, M. N., Fujimoto, M. Y., \& Iben, I., Jr. 2004, ApJ, 611,476

Suda, T., Komiya, Y., Yamada, S., et al. 2013, MNRAS, 432, L46

Tanaka, T., Perna, R., \& Haiman, Z. 2012, MNRAS, 425, 2974

Tegmark, M., Silk, J., Rees, M. J., et al. 1997, ApJ, 474, 1

Tormen, G., Bouchet, F. R., \& White, S. D. M. 1997, MNRAS, 286, 865

Tornatore, L., Ferrara, A., \& Schneider, R. 2007, MNRAS, 382, 945

Turk, M. J., Abel, T., \& O’Shea, B. 2009, Sci, 325, 601

Turk, M. J., Norman, M. L., \& Abel, T. 2010, ApJL, 725, L140

Turk, M. J., Oishi, J. S., Abel, T., \& Bryan, G. L. 2012, ApJ, 745, 154

Valiante, R., Schneider, R., Bianchi, S., \& Andersen, A. C. 2009, MNRAS, 397, 1661

Wada, K., \& Venkatesan, A. 2003, ApJ, 591, 38

Whalen, D., Abel, T., \& Norman, M. L. 2004, ApJ, 610, 14

Wise, J. H., \& Abel, T. 2008, ApJ, 685, 40

Wise, J. H., Turk, M. J., Norman, M. L., \& Abel, T. 2012, ApJ, 745, 50

Wolcott-Green, J., \& Haiman, Z. 2011, MNRAS, 412, 2603

Wolcott-Green, J., Haiman, Z., \& Bryan, G. L. 2011, MNRAS, 418, 838

Yoon, S.-C., Dierks, A., \& Langer, N. 2012, A\&A, 542, A113

Yoshida, N., Abel, T., Hernquist, L., \& Sugiyama, N. 2003, ApJ, 592, 645

Yoshida, N., Omukai, K., \& Hernquist, L. 2008, Sci, 321, 669

Yoshida, N., Omukai, K., Hernquist, L., \& Abel, T. 2006, ApJ, 652, 6 